\title{
Astragalus flavone ameliorates atherosclerosis and hepatic steatosis via improving lipid metabolism and inhibition of inflammation in apoE-/- mice
}

\section{Chuanrui Ma}

First Teaching Hospital of Tianjin University of Traditional Chinese Medicine Jing Zhang

Tianjin University of Traditional Chinese Medicine

Shu Yang

Shenzhen People's Hospital

\section{Yunqing Hua}

First Teaching Hospital of Tianjin University of Traditional Chinese Medicine Jing Su

Tianjin University of Traditional Chinese Medicine

\section{Yuna Shang}

Nankai University College of Life Sciences

\section{Zhongyan Wang}

Nankai University College of Life Sciences

\section{Ke Feng}

Nankai University College of Life Sciences

Jian Zhang

Tianjin Medical University

\section{Hao Zhang}

First Teaching Hospital of Tianjin University of Traditional Chinese Medicine Linna Zhao

First Teaching Hospital of Tianjin University of Traditional Chinese Medicine Jingyuan Mao

First Teaching Hospital of Tianjin University of Traditional Chinese Medicine Guanwei Fan ( $\sim$ guanwei.fan@tjutcm.edu.cn )

Tianjin University of Traditional Chinese medicine

\section{Original investigation}

Keywords: total flavone of Astragalus, cholesterol metabolism, inflammation, miR-33, NFkB, atherosclerosis 
Posted Date: September 2nd, 2020

DOl: https://doi.org/10.21203/rs.3.rs-50056/v1

License: (c) (i) This work is licensed under a Creative Commons Attribution 4.0 International License. Read Full License 


\section{Abstract}

Background: Atherosclerosis is a major pathogenic factor in cardiovascular diseases during the aging process. Foam cell formation and endothelial dysfunction play a key role in the initiation and development of atherosclerosis, which are affected by lipid disorder and inflammation. Therefore, the drug for inhibition of endothelial dysfunction and the subsequent foam cell formation is a therapeutic approach for atherosclerosis treatment. Total flavone of Astragalus membranaceus (TFA) is extracted from Astragalus membranaceus that has protective effect on cardiovascular disease. However, the effect of TFA on atherosclerosis and the underlying mechanism remains unknown.

Methods: In this study, we determined whether TFA could inhibit atherosclerosis; and uncovered the underlying molecular mechanisms. In vivo, apoE deficient mice were treated with TFA contained in highfat diet for 16 weeks. After treatment, aorta, macrophage and serum samples were collected to determine atherosclerotic lesions, lipid metabolism, and expression of associated genes. Concurrently, we investigated the effect of TFA on monocyte adhesion, foam cell formation, endothelial activation, and macrophage polarization in vitro and in vivo.

Results: TFA reduced atherosclerotic plaque size; and enhanced lesion stability by changing the composition of plaque. Moreover, foam cell formation and its accumulation in arterial wall were attenuated by TFA, which might be attributed to improved lipid disorder, reduced inflammation, and decreased monocyte adhesion to endothelial cells. Mechanistically, TFA reduced the expression of scavenger receptor, such as CD36 and SRA; promoted the expression of ATP-binding cassette transporter $A 1$ and $\mathrm{G} 1$ (ABCA1/G1). More importantly, TFA reduced the expression of miR-33, a negative regulator of cholesterol efflux as well as positive mediator on inflammation; and concurrently reduced NFkB activity, by which de-repressed $A B C A 1 / G 1$ activity and inhibited the inflammation.

Conclusions: This study demonstrates that TFA can attenuate atherosclerosis via dual inactivation of miR-33 and NFkB signaling pathway and inhibition of scavenger receptors (CD36 and SRA), which suggests that TFA might be a novel therapeutic approach for inhibition of atherosclerosis.

\section{Introduction}

Atherosclerosis, the principal risk factor of cardiovascular diseases, is mainly driven by vascular lipid deposition and inflammation. Although the introduction of lipid-lowering therapies, such as 3-hydroxy-3methylglutaryl coenzyme A reductase inhibitors and proprotein convertase subtilisin-kexin type 9 inhibitors as well as changes in lifestyle have reduced the risk of cardiovascular disease. The atherosclerosis-induced cardiovascular disease remains the major causes of morbidity and mortality worldwide [1]. Thereby, new strategies to lower the large risk of cardiovascular disease are still needed. Lipid-enriched foam cells are the hallmark of atherosclerotic plaques [2]. Causally, lipid accumulation in the cytoplasm can induce macrophages transform into detrimental foam cells, thereby accelerating 
atherosclerotic plaque destabilization, rupture, and even thrombogenesis [3]. Therefore, inhibition of foam cell formation is a key target for retarding progression of atherosclerosis.

Increased cholesterol uptake and decreased efflux in macrophage can lead to foam cell formation [4], which is mainly determined by the molecules responsible for cholesterol efflux, such as ATP-binding cassette transporter A1 and G1 (ABCA1/G1) [5]; and macrophage scavenger receptors in charge of cholesterol uptake, such as SRA and CD36 [6-8]. Because the transformation of macrophages into foam cells is a driving force in atherosclerosis initiation, stimulation of cellular cholesterol elimination through inhibition of cholesterol uptake or promotion of efflux is a promising antiatherogenic strategy $[9,10]$. Importantly, ABCA1/G1-mediated cholesterol efflux is the initial step of reverse cholesterol transport (RCT) that is defined as the process by which cholesterol moves out of cells in peripheral tissues into the feces, such as foam cells in atherosclerotic plaques.

There is mounting evidence that atherosclerosis is driven not only by dyslipidemia but also by inflammation; and more specifically, chronic inflammation can coordinate with atherogenic lipid dysfunction. Hyperlipidemia can skew plaque macrophages toward an atherogenic inflammatory M1 phenotype instead of toward the antiatherogenic M2 phenotype, by which contributed atherosclerosis development [11]. Recently, a study shown that anti-inflammatory therapy can reduce cardiovascular risk [12]. Importantly, the NF-KB pathway is an important signaling cascade that controls proinflammatory gene expression. In the unstimulated state, the IKB (inhibitor of NF-kB) binds to NF-KB dimers in the cytoplasm to render NF-KB inactive. However, on stimulation by proinflammatory signals, ІкB proteins was phosphorylated by IKB kinases, which leads to IKB degradation and release of active NF-KB dimers for nuclear translocation and target gene induction [13]. In addition, microRNAs (miRNAs) can act as finetuners of gene expression, including genes responsible for lipid metabolism and inflammation, which play important roles in the pathophysiology and progression of atherosclerosis [14]. Among these artherosclerosis-associated miRNAs, elevated levels of miR-33 in foam cell s can limit cholesterol efflux capacity by restricting ABCA1/G1 activity [15]. Therefore, inhibition of miR-33 holds therapeutic promise for atherosclerosis as it has been shown to enhance RCT, raises plasma HDL and lowers VLDL [16-19]. In addition, macrophage polarizaiton and associated inflammation is partially regulated by miR-33 [20]. These results indicate that miR-33 plays dual role in lipid metabolism and inflammation, by which significantly affect the atherosclerosis development.

Flavone has shown the potential to improve HDL function through the effects on cellular antioxidant status and inflammation [21]. Noticeable, the current study showed that higher intake of flavone was associated with a moderately lower risk of developing coronary heart disease [22]. Total flavone of Astragalus membranaceus (TFA) is extracted from Astragalus membranaceus, a traditional Chinese medicine that has protective effects on cardiovascular disease [23, 24]. Additionally, chronic treatment with certain component of Astragalus flavone has shown potential antiatherogenic function. Formononetin, as a component of Astragalus flavone, can protect against obesity that has been defined as an atherogenic contributor [25-27]. Furthermore, formononetin can inhibit foam cell formation [28]; and ameliorate cholestasis through improving cholesterol metabolism [29]. These studies indicate that 
TFA may be atheroprotective. Moreover, compared to the certain component of Astragalus flavone, TFA is easily available in technology and low-cost in economics. Therefore, in this study, we determined whether TFA could attenuate atherosclerosis and attempt to uncover the underlying mechanism. Intriguingly, previous studies have shown that flavone can exert protective effect by modulating miRNAs expression in different model of disease [30-34]. Given the important role of miR-33 in cholesterol metabolism and inflammation, and the key role of NF-KB pathway in inflammation, we further determined whether TFA could inhibit atherosclerosis through regulating miR-33 expression and NF-KB pathway.

In the current study, we demonstrated that TFA reduced atherosclerotic lesion size and enhanced plaque stability in apoE deficient mice, which may be attributed to the improvement of lipid metabolism and inhibition of inflammation. Moreover, TFA suppressed the adhesion of monocytes to inflammatory endothelial cells and promoted macrophage polarization to the M2 phenotype. Mechanistically, these effects may be associated with reduction in miR-33 expression and inactivation of NFKB activity. Collectively, TFA may be a possible therapeutic intervention for atherosclerosis.

\section{Materials And Methods}

\section{Reagents}

Mouse anti-ICAM-1(Cat\#: sc107), VCAM-1(Cat\#: sc13160), GAPDH (Cat\#: sc365062) SRA (Cat\#: sc56777), aSMA (Cat\#: sc130617), CD36 (Cat\#: sc70644), and CD68 (Cat\#: sc20060) monoclonal antibodies were purchased from Santa Cruz Biotechnology, Inc. (Santa Cruz, CA). Rabbit anti-ABCG1 (Cat\#: NB400-132), ABCA1(Cat\#: NB400-105) and SRBI (Cat\#: NB400-104) polyclonal antibodies were purchased from Novus Biologicals (Littleton, CO). Mouse anti-IL-1 $\beta$ (Cat\#: \#12242) monoclonal antibody was purchased from Cell Signaling Technology. Mouse anti-Arg (Cat\#: ab239731) monoclonal antibody were purchased from Abcam (Cambridge, MA). Mouse anti-rabbit IgG-R (Cat\#: sc2492), mouse anti-rabbit IgG-FITC (Cat\#: sc2359) and m-IgGk BP-FITC (Cat\#: sc516140) antibodies were purchased from Santa Cruz Biotechnology, Inc. (Santa Cruz, CA). Astragalus flavone (Cat\#: SA9780) was purchased from Solarbio (Beijing, China).

\section{Cell Culture}

Human umbilical vein endothelial cells (HUVECs) were cultured in VascuLife basal medium containing VEGF lifeFactors Kit (Lifeline Cell Technology, Frederick, MD). RAW264.7, THP-1 cells and peritoneal macrophages were cultured in complete RPMI1640 medium containing 10\% FBS, $50 \mu \mathrm{g} \mathrm{mL}^{-1}$ penicillin/streptomycin and $2 \mathrm{mM}$ glutamine.

\section{Animals And Treatment Schedule}

The protocol for in vivo studies was approved by the Ethics Committee of Tianjin University of Traditional Chinese Medicine and conforms to the Guide for the Care and Use of Laboratory Animals published by the NIH (NIH publication, eighth edition, updated 2011). Eight weeks old, male $\mathrm{ApoE}^{-/-}$mice were purchased from Beijing Vital River Laboratory Animal Technology Co., Ltd. The animals were housed in 
SPF units of the Animal Center at Tianjin University of Traditional Chinese Medicine in the environment with $60-70 \%$ humidity at temperature $22 \pm 1{ }^{\circ} \mathrm{C}$ and $12 \mathrm{~h}$ light-dark cycle. The mice can freely access to water and high-fat diet ( $41 \%$ fat plus $0.5 \%$ cholesterol, MD12015A, Medicience Ltd., China). The ApoE ${ }^{-/-}$ mice were randomly divided into three groups and fed HFD, HFD containing high dose TFA [low dose TFAL,10 mg day ${ }^{-1} \mathrm{~kg}^{-1}$ bodyweight (mpk)], high dose TFA (TFA-L, $20 \mathrm{mpk}$ ) for 16 weeks. The mice were daily checked for food intake, water drink and bodyweight gain during the treatment. At the end of experiment, all mice were anesthetized and euthanized as we previously reported [28, 35], which was followed by collection of aortas, peritoneal macrophages, blood samples and other tissues. Serum was prepared to determine lipid profile, including total cholesterol (Total-C, TC), high-density lipoprotein (HDL)-C, lowdensity lipoprotein (LDL)-C, and triglycerides (TG) [35]. Mousse ox-LDL level was evaluated by the commercially provided ELISA kit.

\section{Atherosclerotic Lesion Analysis}

The aortas were collected and used to prepare aortic root cross sections followed by determination of en face and sinus lesions with Oil Red 0 staining [35]. All the images were obtained with a microscope and quantified lesion areas in en face aorta and aortic root cross sections, respectively. The lesion areas were expressed as $\mu \mathrm{m}^{2}$ or $\%$ of the total surface area. Necrotic core, fibrous cap, cellular apoptosis, collagen content, and expression of CD68, aSMA, Arg1, and IL-1 $\beta$ in lesion within aortic root cross sections were determined by Haematoxylin and eosin (H\&E), sirius red staining, TUNEL staining, and immunofluorescent staining [35]. The vulnerability index of plaques was calculated as previously reported [36].

\section{Determination of foam cell formation in vitro and in vivo}

Foam cell formation in vitro and in vivo was determined by Oil Red 0 staining as described $[28,37]$. In vivo, peritoneal macrophage were isolated from TFA-treated $\mathrm{apoE}^{-/-}$mice and seeded on cover slips in 24-well plates, and then stained with Oil Red 0 solution. In vitro, peritoneal macrophage isolated from untreated $\mathrm{apoE}^{-/-}$mice were seeded on cover slips in 24-well plates. Peritoneal macrophage was incubated with oxLDL for $3 \mathrm{~h}$ following by TFA treatment for $16 \mathrm{~h}$. Cells containing lipid droplets (> $10 /$ cell) were considered as foam cells, and $>10$ fields/sample were counted.

\section{Cholesterol Uptake And Efflux Assay}

Macrophage were incubated in medium containing Dil-oxLDL or 3-dodecanoyl-NBD cholesterol to evaluate ability of cholesterol uptake and efflux as previously studied [28]. Briefly, in 12-well plates, macrophages $\left(1.0 \times 10^{6}\right.$ cells/well) pretreated with TFA at different doses were incubated with $10 \mu \mathrm{g} / \mathrm{mL}$ Dil-oxLDL (Introvegen) in RPMI 1640 at $37^{\circ} \mathrm{C}$ for $6 \mathrm{~h}$. Fluorescence intensity was examined by a fluorescence microscopy. The ability of macrophage cholesterol efflux was determined as we previous study [28]. In brief, macrophage was firstly incubated with 3-dodecanoyl-NBD cholesterol $(1 \mu \mathrm{g} / \mathrm{mL}$, Cayman Chemical) for $6 \mathrm{~h}$. And then cells were switched into serum-free medium containing both apo-Al 
$(5 \mu \mathrm{g} / \mathrm{mL})$ and $\mathrm{HDL}(20 \mu \mathrm{g} / \mathrm{mL})$ as cholesterol acceptor in the presence or absence of TFA for $5 \mathrm{~h}$. The fluorescence-tagged cholesterol in the medium and cells lysate was determined by the automatic microplate reader (Thermo Scientific, Varioskan Lux, USA). Cholesterol efflux was expressed as a ratio of fluorescence in the medium to the total amount of fluorescence in cells together with medium.

\section{Western Blot And Quantitative Real-time PCR}

Total cellular proteins were extracted from cells or liver tissue. Protein expression of ABCA1, ABCG1, CD36, GAPDH, FASN, SREBP1c, SRBI, IKBa, pi-IKBa, p65, pi-p65, and SRA were determined by Western blot as previously reported [38]. Total RNA was isolated and purified, and cDNA was synthesized from $1 \mathrm{mg}$ of total RNA using a reverse transcription kit (Vazyme bioteck co., Itd). For q-RT-PCR, specific genes were amplified by 40 cycles using SYBR green PCR master mix (DBI, Bioscience). Gene-specific primers are listed in Table S1. Expression of mRNA was normalized to the housekeeping gene GAPDH. miR-33 expression was normalized by U6 snRNA expression.

\section{MIR-33 Mimic Transfection In Macrophages}

Macrophages were seeded into plate and cultured to $60 \%$ confluence. Cells were then transfected with miR-33 mimic or miR-33 control (Guangzhou RiboBio Co., Ltd.) using Lipofectamine RNAiMAX (Invitrogen, Grand Island, NE) according to the manufacturer's instructions. The medium was replaced with fresh medium after $6 \mathrm{~h}$ transfection.

\section{Monocyte Adhesion Assay}

The HUVECs were seeded and incubated in 24-well dishes. After reaching $85 \%$ confluence, HUVECs were preincubated with oxLDL $(100 \mu \mathrm{g} / \mathrm{ml})$, or a combination of different dose of TFA $(6,12,24 \mathrm{mg} / \mathrm{ml})$ with oxLDL $(100 \mu \mathrm{g} / \mathrm{ml})$ for $24 \mathrm{~h}$. The THP-1 cells were then added to the 24-well dishes and incubated with HUVECs for $1 \mathrm{~h}$. Subsequently, unbound monocytes were removed by 3 times of washes with warm PBS. After washout, the adherent THP-1 cells to HUVECs were captured with a microscope and counted with ImageJ.

\section{Analysis Of Hepatic Steatosis}

The hepatic steatosis was evaluated as previously described [28, 37, 39]. Briefly, after sacrifice, the liver was isolated ang photographed to exhibit the color and size. In addition, frozen section of liver was prepared to perform HE and Oil red $\mathrm{O}$ staining. Furthermore, Total cholesterol and triglyceride was detected using commercially provided kit.

\section{Statistical analysis}

The data and statistical analysis comply with the recommendations on experimental design and analysis in pharmacology [40]. Data was expressed as means \pm SEM and analyzed by using Graph Pad Prism 
software. One-way ANOVA for comparisons between multiple groups followed by Turkey's method. The value of $\mathrm{p} \otimes 0.05$ was considered statistically significant.

\section{Results}

\section{TFA attenuates atherosclerosis in apo: ${ }^{-/-}$mice}

To test the hypothesis that long-term administration with TFA would protect against atherosclerosis, we treated atherogenic apoE $\mathrm{E}^{-/-}$mice with TFA contained in HFD for 16 weeks. After treatment, we evaluated aortic lesions in apo: $\mathrm{E}^{-/-}$mice. En face analysis of atherosclerosis in the aorta was assessed by Oil red 0 staining and calculated as lesion area or the ratio of lesion area to total area of aorta or aortic root cross sections. Compared with mice fed HFD alone, en face aortic lesions were markedly inhibited by TFA (Fig. 1A and B). Meanwhile, atherosclerotic lesion formation was assessed in four different vascular sites, including the ascending aorta (AA), descending aorta (DA), thoracic aorta (TA), and abdominal aorta (Ad A). The results showed that TFA reduced lesions in the AA, DA, TA, and Ad A (Fig. 1C). Moreover, TFA resulted in significant reduction in the aortic root (Fig. 1D and E). Taken together, the dada suggests that TFA can retard the atherosclerotic lesion development.

\section{TFA Enhances Atherosclerotic Plaque Stability In Apoe Mice}

The high necrotic core size and low fibrous cap area can result in increase of plaque vulnerability even lead to plaque rupture, which can result in myocardial infarction, stroke, and even sudden death. On aortic cross sections, we found necrotic core area within the lesions was significantly smaller while the fibrous cap area was larger in TFA treated mice compared to control mice (Fig. 2A). In addition, TFA increased the collagen positive area (Fig. 2B). Moreover, we observed significant increase in percentages of aSMA ${ }^{+}$ smooth muscle cells and reduction of $\mathrm{CD} 68^{+}$foam cells in TFA group compared to control group (Fig. $2 \mathrm{C}$ and $\mathrm{D}$ ). Dying cells in the plaque can lead to the formation of a prothrombotic necrotic core and vulnerable fibrous cap, a key component of unstable plaques. Therefore, we detected the apoptosis in the plaque by TUNEL staining and observed that TFA significantly reduced the cell apoptosis in situ (Fig. 1E). The quantification of Fig. 2B-E was represented in Fig. 2F. Moreover, the vulnerability index of plaque was reduced by TFA (Fig. 2G). Taken together, the dada suggests that the stability of atherosclerotic plaque can be enhanced by TFA, by which TFA may reduce the risk of plaque rupture and the following cardiovascular events.

\section{TFA inhibits foam cell formation by regulating cholesterol efflux and uptake}

Foam cell is predominant cells in atherosclerotic plaque, inhibition of which can retard the progression of atherosclerosis. In this study, we found that TFA significantly reduced lipid accumulation in peritoneal macrophages from HFD-treated apoE $\mathrm{E}^{-/-}$mice (Fig. 3A and B), suggesting the inhibitory effect of TFA on foam cell formation. In addition, TFA markedly reduced the lipid retention in RAW264.7 cells by Oil red 0 staining (Fig. 3C), which was followed by determination of cellular cholesterol (Fig. 3D). Furthermore, we assessed the capacity of cholesterol uptake and efflux and observed that TFA significantly reduced lipid 
uptake and enhanced the cholesterol efflux (Fig. 3E and F), by which inhibiting the foam cell formation. To delineate the mechanism by which TFA inhibited foam cell formation, we examined the alterations of scavenger receptors (SRA and CD36) and transporters (ABCA1/G1), which are regarded as key mediators in cholesterol homeostasis during foam cell formation. We observed that TFA markedly promoted the ABCA1/G1 expression whereas inhibited the expression of SRA, CD36 (Fig. $3 \mathrm{G}$ and H). We further disclose the mechanism of TFA on ABCA1/G1 expression. Subsequently, we determined whether TFA affected expression of liver $X$ receptors (LXRs), the upstream genes of $A B C A 1 / G 1$, and found that TFA did not affect the expression of $L X R a / \beta$ in transcriptional level (Fig. 3l), indicating that other molecules may mediate the promotive effect of TFA on ABCA1/G1 expression. Moreover, TFA did not change the expression of HMGCR, indicating that TFA did not affect the cholesterol synthesis (Fig. 3I). It is well documented that miR-33 is a post-transcriptional regulator of genes involved in cholesterol homeostasis. Noteworthy, previous study has proven that miR-33 is a negative regulator of cellular ABCA1/G1. To further disclose the mechanism by which TFA stimulate macrophage cholesterol efflux, we determined that whether the TFA-upregulated ABCA1/G1 expression was involved in regulation of miR-33. Intriguingly, TFA significantly reduced the expression of miR-33 (Fig. 3J), and miR-33 mimics treatment almost disrupted the promoting effect of TFA on cholesterol efflux (Fig. S1), indicating that miR-33 plays key role in TFA-mediated inhibitory effect on foam cell formation. Collectively, these results demonstrate that TFA can suppress foam cell formation, mechanistically, through inhibiting scavenger receptormeditated cholesterol uptake and de-repressing miR-33-mediated restriction on cholesterol efflux.

\section{TFA attenuates the inflammatory response in plaque via dual inactivation of NFKB pathway and miR-33 expression}

Inflammation is a key contributor to atherosclerosis development. Therefore, we assessed the expression of proinflammatory cytokines in aorta; and found that TFA significantly reduced the proinflammatory cytokines whereas promoted the anti-inflammatory cytokines (Fig. 4A). We further quantified the levels of representative pro- and anti-inflammatory factors in atherosclerotic lesions and found the mRNA expressions of proinflammatory cytokine IL-1 $\beta$ was downregulated while the anti-inflammatory cytokine Arg1 was upregulated in TFA-treated mice compared with control mice (Fig. 4B), indicating that TFA ameliorates the inflammation during the lesion formation. Macrophages, as the key mediators of inflammatory response, can affect the progression of atherosclerosis. M1 macrophages are present mainly in unstable plaques and can boost the production of pro-atherogenic inflammatory mediators, thereby contributing to sustained inflammation and plaque vulnerability. Therefore, we further determined the effect of TFA on macrophage polarization and observed that the peritoneal macrophage from TFAtreated mice are prone to $\mathrm{M} 2$ transition but not $\mathrm{M} 1$ polarization (Fig. $4 \mathrm{C}$ and $\mathrm{D}$ ). To uncover the underlying mechanism of anti-inflammatory effect by TFA, we assessed NFKB pathway, the key regulator of inflammation. Noticeable, TFA significantly increased the expression of IкBa and reduced the phosphorylation of IKBa and p65 (Fig. 4E), indicating that NFKB pathway was markedly inactivated and thereby the inflammation was ameliorated. Moreover, miR-33 is a post-transcriptional regulator of genes involved in inflammation, and inhibition of which can reduce plaque macrophage inflammation [41]. Intriguingly, in peritoneal macrophage isolated from TFA-treated mice, expression of miR-33 was 
markedly reduced (Fig. 4F), indicating that the anti-inflammatory effect of TFA may be associated with reduction of miR-33 expression. Taken together, we demonstrate that TFA reduced inflammation in vivo through inactivation of NFKB and negative regulation of miR-33 expression, by which contributing to its anti-atherogenic function.

\section{TFA promotes macrophage phenotypic transition to M2 anti-inflammatory type in vitro}

We further detected effect of TFA on the inflammatory factor generation in vitro macrophage by immunofluorescent staining and q-RT-PCR. The proinflammatory cytokines IL1 $\beta$ and TNFa were downregulated, whereas the anti-inflammatory cytokines Arg1 and IL10 were upregulated (Fig. 5A and B). To uncover the underlying mechanism of anti-inflammatory effect by TFA, we assessed NFKB pathway in vitro as we did in vivo. Indeed, TFA significantly reduced the activity of NFKB pathway (Fig. 5C). Moreover, we further determined whether the anti-inflammatory effect of TFA was associated miR-33. We have shown that TFA significantly reduced miR-33 expression in vitro and in vivo (Fig. 3J and Fig. 4F), which partially accounted for the anti-inflammatory effect of TFA. Furthermore, we treated macrophages with TFA in presence or absence of miR-33 mimics. Noticeable, TFA promoted the macrophage toward M2 phenotype, however, which was abolished by miR-33 mimics (Fig. 5D), indicating that the regulatory effect on macrophage phenotypic transition is miR-33-involved. Taken together, TFA can inhibit inflammatory in vitro, which was associated inactivation of NFKB pathway and negative regulation of miR-33 expression.

\section{TFA inhibits the endothelial activation and monocyte recruitment to endothelial cells}

Circulating monocytes are recruited by inflammatory cytokines to the endothelium in the aorta, differentiate into macrophages, subsequently transform into foam cells under LDL or oxLDL stimulation, and thereby contributing aortic lesions formation. Therefore, we determined whether TFA could inhibit the monocyte adhesion to endothelial cells (ECs). As shown in Fig. 6A and B, TFA significantly reduced the number of THP-1 cells adhering to HUVECs. ECs actively participate in the regulatory process of leukocytes trans-endothelial migration during vascular inflammation. Moreover, adhesion molecules from ECs bind to specific ligands expressed by monocytes, such as CD36 and SRA, resulting in the increased leukocyte-endothelial interactions [42, 43]. Mechanistically, we detected the effect of TFA on expression of ICAM-1 and VCAM-1 in HUVEC and observed that both were significantly reduced (Fig. 6C). Proinflammatory cytokines can induce the expression of adhesion molecules in ECs, such as ICAM- 1 and VCAM-1, which provide a scaffold for leukocyte migration in ECs. Moreover, in THP-1 cells, TFA markedly reduced expression of proinflammatory cytokines, including TNFa, IL1 $\beta$, and IL-6 (Fig. 6F), through which leading to inactivation of the ICAM-1 and VCAM-1 expression in HUVEC. Furthermore, we assessed the expression of CD36 and SRA in THP-1 cells and found that both scavenger receptors were reduced by TFA (Fig. 6E), indicating that the monocyte binding ligand was reduced, which contributed the reduction in monocyte adhesion to HUVEC. Collectively, TFA reduced monocyte adhesion to endothelial cells by reducing binding ligands (CD36 and SRA) in monocyte and inflammation-induced adhesion molecules (ICAM-1 and VCAM-1) expression in ECs, partially by which exerted the antiatherogenic function. 


\section{TFA Improves The HFD-induced Dyslipidemia In Apoe Mice}

Lipid dysfunction is a critical contributor to atherosclerosis development. The influx of LDL into the arterial intima, the site of atherogenesis, is closely associated with their plasma concentration because high concentrations of LDL lead to higher LDL uptake by macrophages. In addition, infiltrated LDL are oxidized to turn into highly atherogenic forms, such as ox-LDL. Macrophages ingest the modified LDL particles via scavenger receptors and thereby transformed into foam cells. In contrast, HDL is considered antiatherogenic lipoproteins because it can promote cholesterol efflux from foam cells, initiating the RCT, which involves the transfer of cholesterol to the liver and, ultimately, to the gut for excretion. In this study, we detected whether the serum lipid profile was improved by TFA. The body weight was not changed by TFA (Fig. 7A). However, TFA significantly reduced level of total cholesterol (T-CHO), LDL, TG, and VLDL, whereas increased the HDL level (Fig. 7B-E). We further quantified the level of ox-LDL, a modified LDL that contributed to atherosclerosis development, and observed that ox-LDL was downregulated in TFAtreated mice compared with control mice (Fig. 7F), suggesting that the atherogenic form of LDL was reduced and oxidant stress may be attenuated by TFA. Collectively, TFA improved the lipid profile and restrained the transformation of LDL to atherogenic form during the atherosclerosis development, partially by which exerted the anti-atherogenic function.

\section{TFA Ameliorates The HFD-induced Hepatic Steatosis In Apoe Mice}

As shown in Fig. 8A, liver color and weight were changed to almost normal condition, which was followed by reduced ratio of liver weight to body weight (Fig. 8B). Moreover, H\&E and Oil Red O staining revealed that hepatic steatosis was attenuated in TFA-treated mice compared to control mice after administration of HFD (Fig. 8C and 8F). Furthermore, TFA significantly reduced the hepatic TC and TG content (Fig. 8D and E), indicating that hepatic steatosis was attenuated by TFA. Hepatic steatosis can lead to the liver injury. Noticeable, chronic treatment with TFA did not cause toxicity as measured by plasma AST and ALT enzyme levels. In contrast, TFA markedly reduced the HFD-induced liver injury, which was shown by the reduced levels of AST and ALT (Fig. 8G and H). To further determine the mechanism of TFA on liver metabolism, we assessed whether the expression of genes involved lipid oxidation and genesis were changed by TFA. Intriguingly, TFA markedly promoted the expression of SRBI, the gene in charge of cholesterol uptake in liver; and simultaneously promoted the expression of ABCG5/G8, the genes responsible for cholesterol transportation to intestine (Fig. $8 \mathrm{I}$ and $\mathrm{J}$ ), by which reduced the hepatic retention of cholesterol and enhanced the RCT. In addition, TFA significantly reduced expression of genes responsible for lipid genesis, including FASN and SREBP1c (Fig. 8K), indicating that TFA inhibited lipid synthesis. Consistent with this observation, the protein or mRNA expression of genes involved in fatty acid oxidation (AMPKa and CPT1a) were upregulated in TFA treated mice compared to control mice after HFD feeding (Fig. 8L-M), which indicated that TFA promoted the lipid consumption. Taken together, TFA attenuated the hepatic steatosis by promoting RCT, enhancing fatty acid oxidation, and downregulating lipid synthesis, by which improved the lipid metabolism and thereby partially account for its antiatherogenic function. 


\section{Discussion}

Atherosclerosis, a major pathogen of coronary heart disease (CHD), is closely associated with lipid disorder and chronic inflammation. TFA is the flavone component from Astragalus membranaceus, the long-term used traditional Chinese medicine for CHD treatment in clinic. Noticeable, the current study showed that higher intake of flavone was associated with a lower risk of developing CHD [22]. In our previous study, we found that the formononetin, a kind of flavone, has atheroprotective effect [28]. In this study, we determined whether TFA could inhibit atherosclerosis, and attempted to uncover the underlying mechanism. Intriguingly, we found that TFA treatment substantially reduced the atherosclerotic development, enhanced the plaque stability, and reduced endothelial activation and monocyte recruitment, which may be attributed to the effect of improving the lipid disorder and anti-inflammation. Mechanistically, we found that miR-33 is a critical signaling mediator of TFA on promoting expression of ABCA1/G1 as well as inactivation of proinflammatory gene expression in macrophages, which coordinated the anti-inflammatory effect of TFA through inactivating NFKB signaling pathway.

Increased vulnerability is prone to rupture of atherosclerotic plaque, which can result in severe cardiovascular event [44]. Of note, composition of plaque markedly affect the lesion stability [45]. In this study, TFA significantly reduced atherosclerotic plaque size (Fig. 1) and promoted a more favorable plaque composition with increased fibrous cap area, plaque collagen and SMC content; and reduced necrotic core area and macrophage accumulation (Fig. 2), indicating that TFA significantly reduced the plaque vulnerability. Moreover, we found that TFA markedly promotes the expression of ABCA1/G1 and inhibits the CD36 and SRA expression (Fig. $3 G$ and H), by which enhanced the cholesterol efflux, reduced the cholesterol uptake and thereby inhibited foam cell formation. However, as the upstream regulatory gene of ABCA1/G1, liver X receptor (LXR) was not activated by TFA (Fig. 3I). Moreover, the key gene for cholesterol synthesis, HMGCR, was also not affected by TFA (Fig. 3I). These results indicated that other molecule may mediate the TFA-upregulated expression of transporters.

To gain further insight into the effect of TFA on ABCA1/G1 expression, we investigated the mechanism underlying the effect of TFA on cholesterol efflux. Noticeable, miRNAs, a group of small endogenous noncoding RNAs, can regulate gene expression at posttranscriptional levels. Functionally, miRNAs can serve as important regulators of atherogenic process, such as cellular adhesion, lipid uptake and efflux, and generation of inflammatory mediators [14]. Therefore, we hypothesized that miRNAs may mediate the protective effect of TFA on lipid metabolism and inflammation, thereby inhibiting the atherosclerosis development. Noticeable, miR-33 is a negative regulator of ABCA1/G1 and thereby promoting foam cell formation by inhibiting macrophage cholesterol efflux [17]. Therefore, we further postulated that the enhancement of $A B C A 1 / G 1$ and the inhibition of inflammation by TFA may be through suppression of miR-33, by which remove the restriction on expression of ABCA1/G1. To test the hypothesis, we evaluated miR-33 expression in vitro and in vivo and found that miR-33 was reduced by TFA (Fig. $3 \mathrm{~J}$ ), which account for the upregulation of ABCA1/G1 expression. 
Atherosclerosis is characterized with the predominance of an M1 macrophage phenotype within the plaque, whereas plaques undergoing regression are enriched in $M 2$ macrophages [46, 47]. Intriguingly, in this study, TFA significantly inhibited M1 macrophage polarization, whereas promoted M2 macrophage phenotype in vivo and in vitro, as indicated by the reduced levels of proinflammatory cytokines and increased anti-inflammatory cytokines (Fig. 4A-D and Fig. 5A). NFKB signaling pathway plays important role in regulating macrophage polarization and associated inflammation [48]. In addition, miR-33 can sustain the inflammatory M1-like macrophage phenotype and inhibition of which can reduce plaque macrophage inflammation $[20,41]$. Therefore, we determined whether the anti-inflammatory effect of TFA was involved in NFKB and miR-33. We assessed not only the activity of NFKB pathway but also the expression of miR-33 and observed that TFA markedly inactivated the NFKB activity (Fig. 4E and Fig. 5C) and reduced the expression of miR-33 (Fig. 4F). Intriguingly, miR-33 mimic almost abolished the effect of TFA on macrophage inflammation (Fig. 5D). These results indicated that anti-inflammatory effect of TFA may be attributed to dual suppression of miR-33 and NFKB pathway, which may account for TFA-induced the reduction in pro-inflammatory cytokines in macrophages and aortas (Fig. 4A and D), and ICAM-1 and VCAM-1 expression in ECs (Fig. 6C and D).

Atherogenic endothelial activation enhances adhesiveness for circulating monocytes, and especially in the context of hypercholesterolemia, these monocytes accumulate excessive lipid to drive early plaque formation [49]. In the monocyte attachment assay, TFA markedly suppressed the monocyte recruitment to ECs, and mechanistically reduced the expression of VCAM-1 and ICAM-1 (Fig. 6A-D), the major molecules that expressed in the activated ECs [50], indicating that TFA can attenuate the endothelial activation and the following monocyte adhesion. Moreover, TFA significantly reduced the HFD-induced the lipid disorder (Fig. 7A-F), which favored the reduction in cellular lipid accumulation in monocyte and thereby retarded the initiation and development of atherosclerosis.

Liver is a critical organ that regulate lipid metabolism, which mainly determines the cholesterol metabolism and lipid profile in serum, thereby affecting the atherogenesis. Despite no significant differences were observed in body weight over the 16 weeks of HFD along with TFA feeding, the serum lipid profile was significantly improved by TFA (Fig. 7). SREBP-1c can activate transcription of genes involved in lipid synthesis, such as fatty acid synthase (FASN) [51]. Noteworthy, in the liver, TFA markedly damped the expression of several lipogenic genes, such as SREBP-1c and FASN while increased the expression of CPT1 $\mathrm{a}$ and AMPKa (Fig. 8K, L and M), indicating that lipid synthesis decreased, and fatty acid oxidation (FAO) increased. Noticeable, miR-33, an intronic miRNA that co-expressed with its host gene SREBP1 [52], can balance cellular lipid levels by increasing genes that oppose SREBP-regulated pathways, including those involved in cholesterol efflux and FAO. For instance, in non-human primate model, miR-33 antagonism increased FAO and reduced fatty acid synthesis [19]. In this study, hepatic miR-33 expression was reduced by TFA (Fig.S2), which may be attributed to repression of SREBP1 (Fig. 8K). In addition, scavenger receptor class B member 1 (SR-BI) is key molecule for RCT, loss of which in people are associated with increased cardiovascular risk [53]. After ABCA1/G1-mediated cholesterol efflux to HDL, mature HDL can directly deliver cholesterol to the liver via SR-BI, by which mediates RCT and are thereby anti-atherogenic [54-56]. In this study, we found that TFA significantly enhanced SRBI 
expression (Fig. $8 \mathrm{I}$ and K), which may promote RCT. Additionally, the TFA-induced increase in efflux capacity was significantly correlated with plasma HDL concentrations (Fig. 7C). Moreover, the biliary cholesterol excretion rate is linearly related to the expression of ABCG5/G8 [57, 58]; and it should be noted that the RCT rate in the mice can be promoted by increasing the hepatic expression of ABCG5/G8 [59]. In the present study, TFA markedly increased the expression of the ABCG5/G8 (Fig. 8J), indicating that TFA may contribute to the cholesterol excretion into bile and thereby increasing RCT.

\section{Conclusions}

Taken together, TFA can attenuate the atherosclerosis development via inhibiting foam cell formation and inflammation, which is through negative regulation of miR-33, CD36, and SRA expression; and inactivation of NF-KB pathway. Simultaneously, TFA markedly ameliorate hepatic steatosis via improving the hepatic lipid metabolism and thereby reducing proatherogenic lipid disorder, which is through promoting reverse cholesterol transport, enhancing fatty acid oxidation, and downregulating lipid synthesis. Therefore, TFA may act as a very promising drug for atherosclerosis and hepatic steatosis treatment.

\section{List Of Abbreviations}

Arg1, arginase 1; CPT1a, carnitine palmitoyl transferase 1 alpha; CXCL1, C-X-C motif chemokine ligand 1; IFN- $y$, Interferon gamma; Mgl2, macrophage lectin 2; Mrc1, mannose receptor C-type 1; NOS2, nitric oxide synthase 2; SREBP1c, sterol regulatory element-binding protein 1c; TGF- $\beta 1$, transforming growth factor $\beta 1 ; F A S N$, fatty acid synthase; SR-BI, scavenger receptor class $B$ type $1 ; R C T$, reverse cholesterol transport. IL, interleukin; TNFa, tumor necrosis factor a; iNOS, inducible nitric oxide synthase; GAPDH, glyceraldehyde-3-phosphate dehydrogenase; COX2, prostaglandin-endoperoxide synthase 2; TLR4, toll like receptor 4; MCP-1, monocyte chemotactic protein 1; NLRP3, NLR family pyrin domain containing 3; VCAM-1, vascular cell adhesion molecule 1; ICAM-1, intercellular adhesion molecule 1; PECAM1, platelet and endothelial cell adhesion molecule 1; NF-kB, nuclear factor kappa B; CCL, C-C motif chemokine ligand; eNOS, endothelia NO synthase; PPARy, peroxisome proliferator activated receptor gamma; Chi3I3, chitinase-like 3; FOXP3, fork head box P3; VEGF, vascular endothelial growth factor; MMP, matrix metallopeptidase; MERTK, MER proto-oncogene tyrosine kinase; HMGCR, 3-hydroxy-3-methylglutaryl-CoA reductase; $L X R a / \beta$, Liver $X$ receptor $\alpha / \beta$

\section{Declarations}

\section{Ethics approval and consent to participate}

The protocol for in vivo studies was approved by the Ethics Committee of Tianjin University of Traditional Chinese Medicine and conforms to the Guide for the Care and Use of Laboratory Animals published by the NIH (NIH publication, eighth edition, updated 2011). 


\section{Consent for publication}

Not applicable

\section{Availability of data and materials}

All data are provided and available in this manuscript.

\section{Competing interests}

The authors declare that they have no competing interests

\section{Funding}

This work was supported by grants from National Major Scientific and Technological Special Project for "Significant New Drugs Development" (2019ZX09201005-007-001); National Natural Science Foundation of China (81774050); Tianjin Outstanding Youth Science Foundation (17JCJQJC46200); Natural Science Foundation of Tianjin (17JCYBJC29000, 19JCQNJC12600); Research project of Tianjin education commission (2019KJ044); Training Program Foundation for Innovative Research Team of Higher Education in Tianjin during the 13th Five-Year Plan Period (NO.TD13-5050)

\section{Authors' contributions}

Chuanrui Ma wrote the article. Jing Zhang, Shu Yang, Yunqing Hua, Hao Zhang, and Linna Zhao conducted the experiment. Jing Su, Yuna Shang, Zhongyan Wang, Ke Feng, and Jian Zhang provided advices. Jingyuan Mao and Guanwei Fan designed the experiment. All authors read and approved the final manuscript.

\section{Acknowledgments}

Not applicable

\section{References}

1. de Winther MPJ, Lutgens E: The Link between Hematopoiesis and Atherosclerosis. N Engl J Med 2019, 380(19):1869-1871.

2. Moore KJ, Tabas I: Macrophages in the pathogenesis of atherosclerosis. Cell 2011, 145(3):341-355.

3. Yang Y, Li X, Peng L, An L, Sun N, Hu X, Zhou P, Xu Y, Li P, Chen J: Tanshindiol C inhibits oxidized lowdensity lipoprotein induced macrophage foam cell formation via a peroxiredoxin 1 dependent pathway. Biochim Biophys Acta Mol Basis Dis 2018, 1864(3):882-890.

4. Yu XH, Fu YC, Zhang DW, Yin K, Tang CK: Foam cells in atherosclerosis. Clinica chimica acta; international journal of clinical chemistry 2013, 424:245-252. 
5. Tall AR, Yvan-Charvet L, Terasaka N, Pagler T, Wang N: HDL, ABC transporters, and cholesterol efflux: implications for the treatment of atherosclerosis. Cell metabolism 2008, 7(5):365-375.

6. Crucet M, Wust SJ, Spielmann P, Luscher TF, Wenger RH, Matter CM: Hypoxia enhances lipid uptake in macrophages: role of the scavenger receptors Lox1, SRA, and CD36. Atherosclerosis 2013, 229(1):110-117.

7. Nakata A, Nakagawa Y, Nishida M, Nozaki S, Miyagawa J, Nakagawa T, Tamura R, Matsumoto K, Kameda-Takemura K, Yamashita S et al: CD36, a novel receptor for oxidized low-density lipoproteins, is highly expressed on lipid-laden macrophages in human atherosclerotic aorta. Arteriosclerosis, thrombosis, and vascular biology 1999, 19(5):1333-1339.

8. de Winther MP, van Dijk KW, Havekes LM, Hofker MH: Macrophage scavenger receptor class A: A multifunctional receptor in atherosclerosis. Arteriosclerosis, thrombosis, and vascular biology 2000, 20(2):290-297.

9. Tall AR: Plasma high density lipoproteins: Therapeutic targeting and links to atherogenic inflammation. Atherosclerosis 2018, 276:39-43.

10. Ouimet M, Barrett TJ, Fisher EA: HDL and Reverse Cholesterol Transport. Circ Res 2019, 124(10):1505-1518.

11. Barrett TJ, Distel E, Murphy AJ, Hu J, Garshick MS, Ogando Y, Liu J, Vaisar T, Heinecke JW, Berger JS et al: Apolipoprotein Al) Promotes Atherosclerosis Regression in Diabetic Mice by Suppressing Myelopoiesis and Plaque Inflammation. Circulation 2019, 140(14):1170-1184.

12. Lutgens E, Atzler D, Döring Y, Duchene J, Steffens S, Weber C: Immunotherapy for cardiovascular disease. Eur Heart J 2019, 40(48):3937-3946.

13. Baeuerle PA, Baltimore D: I kappa B: a specific inhibitor of the NF-kappa B transcription factor. Science 1988, 242(4878):540-546.

14. Feinberg MW, Moore KJ: MicroRNA Regulation of Atherosclerosis. Circ Res 2016, 118(4):703-720.

15. Price NL, Rotllan N, Zhang X, Canfrán-Duque A, Nottoli T, Suarez Y, Fernández-Hernando C: Specific Disruption of Abca1 Targeting Largely Mimics the Effects of miR-33 Knockout on Macrophage Cholesterol Efflux and Atherosclerotic Plaque Development. Circ Res 2019, 124(6):874-880.

16. Rayner KJ, Suárez Y, Dávalos A, Parathath S, Fitzgerald ML, Tamehiro N, Fisher EA, Moore KJ, Fernández-Hernando C: MiR-33 contributes to the regulation of cholesterol homeostasis. Science 2010, 328(5985):1570-1573.

17. Rayner KJ, Sheedy FJ, Esau CC, Hussain FN, Temel RE, Parathath S, van Gils JM, Rayner AJ, Chang AN, Suarez $Y$ et al: Antagonism of miR-33 in mice promotes reverse cholesterol transport and regression of atherosclerosis. J Clin Invest 2011, 121(7):2921-2931.

18. Horie T, Baba O, Kuwabara Y, Chujo Y, Watanabe S, Kinoshita M, Horiguchi M, Nakamura T, Chonabayashi $\mathrm{K}$, Hishizawa $\mathrm{M}$ et al: MicroRNA-33 deficiency reduces the progression of atherosclerotic plaque in ApoE-/- mice. J Am Heart Assoc 2012, 1(6):e003376.

19. Rayner KJ, Esau CC, Hussain FN, McDaniel AL, Marshall SM, van Gils JM, Ray TD, Sheedy FJ, Goedeke L, Liu X et al: Inhibition of miR-33a/b in non-human primates raises plasma HDL and lowers 
VLDL triglycerides. Nature 2011, 478(7369):404-407.

20. Ouimet M, Ediriweera HN, Gundra UM, Sheedy FJ, Ramkhelawon B, Hutchison SB, Rinehold K, van Solingen C, Fullerton MD, Cecchini K et al: MicroRNA-33-dependent regulation of macrophage metabolism directs immune cell polarization in atherosclerosis. J Clin Invest 2015, 125(12):43344348.

21. Millar CL, Duclos Q, Blesso CN: Effects of Dietary Flavonoids on Reverse Cholesterol Transport, HDL Metabolism, and HDL Function. Adv Nutr 2017, 8(2):226-239.

22. Ma L, Liu G, Ding M, Zong G, Hu FB, Willett WC, Rimm EB, Manson JE, Sun Q: Isoflavone Intake and the Risk of Coronary Heart Disease in US Men and Women: Results From 3 Prospective Cohort Studies. Circulation 2020, 141(14):1127-1137.

23. Liu CH, Tsai CH, Li TC, Yang YW, Huang WS, Lu MK, Tseng CH, Huang HC, Chen KF, Hsu TS et al: Effects of the traditional Chinese herb Astragalus membranaceus in patients with poststroke fatigue: A double-blind, randomized, controlled preliminary study. Journal of ethnopharmacology 2016, 194:954-962.

24. Su D, Li HY, Yan HR, Liu PF, Zhang L, Cheng JH: Astragalus Improved Cardiac Function of Adriamycin-Injured Rat Hearts by Upregulation of SERCA2a Expression. The American journal of Chinese medicine 2009, 37(3):519-529.

25. Lovren F, Teoh H, Verma S: Obesity and atherosclerosis: mechanistic insights. The Canadian journal of cardiology 2015, 31(2):177-183.

26. Gautam J, Khedgikar V, Kushwaha P, Choudhary D, Nagar GK, Dev K, Dixit P, Singh D, Maurya R, Trivedi R: Formononetin, an isoflavone, activates AMP-activated protein kinase/beta-catenin signalling to inhibit adipogenesis and rescues C57BL/6 mice from high-fat diet-induced obesity and bone loss. The British journal of nutrition 2017, 117(5):645-661.

27. Nie T, Zhao S, Mao L, Yang Y, Sun W, Lin X, Liu S, Li K, Sun Y, Li P et al: The natural compound, formononetin, extracted from Astragalus membranaceus increases adipocyte thermogenesis by modulating PPARgamma activity. Br J Pharmaco/2018, 175(9):1439-1450.

28. Ma C, Xia R, Yang S, Liu L, Zhang J, Feng K, Shang Y, Qu J, Li L, Chen N et al: Formononetin attenuates atherosclerosis via regulating interaction between KLF4 and SRA in apoE(-/-) mice. Theranostics 2020, 10(3):1090-1106.

29. Yang S, Wei L, Xia R, Liu L, Chen Y, Zhang W, Li Q, Feng K, Yu M, Zhang W et al: Formononetin ameliorates cholestasis by regulating hepatic SIRT1 and PPARa. Biochem Biophys Res Commun 2019, 512(4):770-778.

30. Huang Z, Huang Q, Ji L, Wang Y, Qi X, Liu L, Liu Z, Lu L: Epigenetic regulation of active Chinese herbal components for cancer prevention and treatment: A follow-up review. Pharmacol Res 2016, 114:1-12.

31. Rigalli JP, Tocchetti GN, Arana MR, Villanueva SS, Catania VA, Theile D, Ruiz ML, Weiss J: The phytoestrogen genistein enhances multidrug resistance in breast cancer cell lines by translational regulation of ABC transporters. Cancer Lett 2016, 376(1):165-172. 
32. Sun DW, Zhang HD, Mao L, Mao CF, Chen W, Cui M, Ma R, Cao HX, Jing CW, Wang Z et al: Luteolin Inhibits Breast Cancer Development and Progression In Vitro and In Vivo by Suppressing Notch Signaling and Regulating MiRNAs. Cell Physiol Biochem 2015, 37(5):1693-1711.

33. Yin M, Yuan Y, Cui Y, Hong X, Luo H, Hu X, Tang M, Hescheler J, Xi J: Puerarin Suppresses the SelfRenewal of Murine Embryonic Stem Cells by Inhibition of REST-MiR-21 Regulatory Pathway. Cell Physiol Biochem 2015, 37(2):527-536.

34. Lam TK, Shao S, Zhao Y, Marincola F, Pesatori A, Bertazzi PA, Caporaso NE, Wang E, Landi MT: Influence of quercetin-rich food intake on microRNA expression in lung cancer tissues. Cancer Epidemiol Biomarkers Prev 2012, 21(12):2176-2184.

35. Ma C, Zhang W, Yang X, Liu Y, Liu L, Feng K, Zhang X, Yang S, Sun L, Yu M et al: Functional interplay between liver $X$ receptor and AMP-activated protein kinase alpha inhibits atherosclerosis in apolipoprotein E-deficient mice - a new anti-atherogenic strategy. Br J Pharmaco/2018, 175(9):14861503.

36. Williams H, Johnson JL, Carson KG, Jackson CL: Characteristics of intact and ruptured atherosclerotic plaques in brachiocephalic arteries of apolipoprotein E knockout mice. Arterioscler Thromb Vasc Biol 2002, 22(5):788-792.

37. Ma C, Zhang W, Yang X, Liu Y, Liu L, Feng K, Zhang X, Yang S, Sun L, Yu M et al: Functional interplay between liver $X$ receptor and AMP-activated protein kinase $a$ inhibits atherosclerosis in apolipoprotein E-deficient mice - a new anti-atherogenic strategy. Br J Pharmacol 2018, 175(9):14861503.

38. Yang S, Ma C, Wu H, Zhang H, Yuan F, Yang G, Yang Q, Jia L, Liang Z, Kang L: Tectorigenin attenuates diabetic nephropathy by improving vascular endothelium dysfunction through activating AdipoR1/2 pathway. Pharmacol Res 2020, 153:104678.

39. Wang Z, Ma C, Shang Y, Yang L, Zhang J, Yang C, Ren C, Liu J, Fan G, Liu J: Simultaneous coassembly of fenofibrate and ketoprofen peptide for the dual-targeted treatment of nonalcoholic fatty liver disease (NAFLD). Chem Commun (Camb) 2020, 56(36):4922-4925.

40. Curtis MJ, Bond RA, Spina D, Ahluwalia A, Alexander SP, Giembycz MA, Gilchrist A, Hoyer D, Insel PA, Izzo AA et al: Experimental design and analysis and their reporting: new guidance for publication in BJP. Br J Pharmacol 2015, 172(14):3461-3471.

41. Distel E, Barrett TJ, Chung K, Girgis NM, Parathath S, Essau CC, Murphy AJ, Moore KJ, Fisher EA: miR33 inhibition overcomes deleterious effects of diabetes mellitus on atherosclerosis plaque regression in mice. Circ Res 2014, 115(9):759-769.

42. Trinh-Trang-Tan MM, Vilela-Lamego C, Picot J, Wautier MP, Cartron JP: Intercellular adhesion molecule-4 and CD36 are implicated in the abnormal adhesiveness of sickle cell SAD mouse erythrocytes to endothelium. Haematologica 2010, 95(5):730-737.

43. Santiago-García J, Kodama T, Pitas RE: The class A scavenger receptor binds to proteoglycans and mediates adhesion of macrophages to the extracellular matrix. J Biol Chem 2003, 278(9):6942-6946. 
44. Seneviratne AN, Edsfeldt A, Cole JE, Kassiteridi C, Swart M, Park I, Green P, Khoyratty T, Saliba D, Goddard ME et al: Interferon Regulatory Factor 5 Controls Necrotic Core Formation in Atherosclerotic Lesions by Impairing Efferocytosis. Circulation 2017, 136(12):1140-1154.

45. Naghavi M, Libby P, Falk E, Casscells SW, Litovsky S, Rumberger J, Badimon JJ, Stefanadis C, Moreno P, Pasterkamp G et al: From vulnerable plaque to vulnerable patient: a call for new definitions and risk assessment strategies: Part II. Circulation 2003, 108(15):1772-1778.

46. Lutgens E, Atzler D, Doring Y, Duchene J, Steffens S, Weber C: Immunotherapy for cardiovascular disease. European heart journal 2019.

47. Tunon J, Badimon L, Bochaton-Piallat ML, Cariou B, Daemen MJ, Egido J, Evans PC, Hoefer IE, Ketelhuth DFJ, Lutgens $\mathrm{E}$ et al: Identifying the anti-inflammatory response to lipid lowering therapy: a position paper from the working group on atherosclerosis and vascular biology of the European Society of Cardiology. Cardiovascular research 2019, 115(1):10-19.

48. Song W, Zhang CL, Gou L, He L, Gong YY, Qu D, Zhao L, Jin N, Chan TF, Wang L et al: Endothelial TFEB (Transcription Factor EB) Restrains IKK (IKB Kinase)-p65 Pathway to Attenuate Vascular Inflammation in Diabetic db/db Mice. Arterioscler Thromb Vasc Biol 2019, 39(4):719-730.

49. Tabas I, García-Cardeña G, Owens GK: Recent insights into the cellular biology of atherosclerosis. J Cell Biol 2015, 209(1):13-22.

50. Gerhardt T, Ley K: Monocyte trafficking across the vessel wall. Cardiovascular research 2015, 107(3):321-330.

51. Shimano H: SREBPs: physiology and pathophysiology of the SREBP family. Febs j 2009, 276(3):616621.

52. Najafi-Shoushtari SH, Kristo F, Li Y, Shioda T, Cohen DE, Gerszten RE, Näär AM: MicroRNA-33 and the SREBP host genes cooperate to control cholesterol homeostasis. Science 2010, 328(5985):15661569.

53. Zanoni P, Khetarpal SA, Larach DB, Hancock-Cerutti WF, Millar JS, Cuchel M, DerOhannessian S, Kontush A, Surendran P, Saleheen D et al: Rare variant in scavenger receptor BI raises HDL cholesterol and increases risk of coronary heart disease. Science 2016, 351(6278):1166-1171.

54. Shen WJ, Azhar S, Kraemer FB: SR-B1: A Unique Multifunctional Receptor for Cholesterol Influx and Efflux. Annu Rev Physiol 2018, 80:95-116.

55. Rader DJ, Alexander ET, Weibel GL, Billheimer J, Rothblat GH: The role of reverse cholesterol transport in animals and humans and relationship to atherosclerosis. J Lipid Res 2009, 50 Suppl(Suppl):S189-194.

56. Rosenson RS, Brewer HB, Jr., Davidson WS, Fayad ZA, Fuster V, Goldstein J, Hellerstein M, Jiang XC, Phillips MC, Rader DJ et al: Cholesterol efflux and atheroprotection: advancing the concept of reverse cholesterol transport. Circulation 2012, 125(15):1905-1919.

57. Nijstad N, Gautier T, Briand F, Rader DJ, Tietge UJ: Biliary sterol secretion is required for functional in vivo reverse cholesterol transport in mice. Gastroenterology 2011, 140(3):1043-1051. 
58. Lee-Rueckert M, Escola-Gil JC, Kovanen PT: HDL functionality in reverse cholesterol transportChallenges in translating data emerging from mouse models to human disease. Biochim Biophys Acta 2016, 1861(7):566-583.

59. Escolà-Gil JC, Llaverias G, Julve J, Jauhiainen M, Méndez-González J, Blanco-Vaca F: The cholesterol content of Western diets plays a major role in the paradoxical increase in high-density lipoprotein cholesterol and upregulates the macrophage reverse cholesterol transport pathway. Arterioscler Thromb Vasc Biol 2011, 31(11):2493-2499.

\section{Figures}

Ma et al,Figure 1

A



TFA-L



C

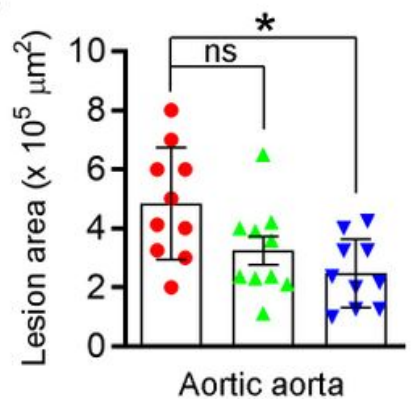

D
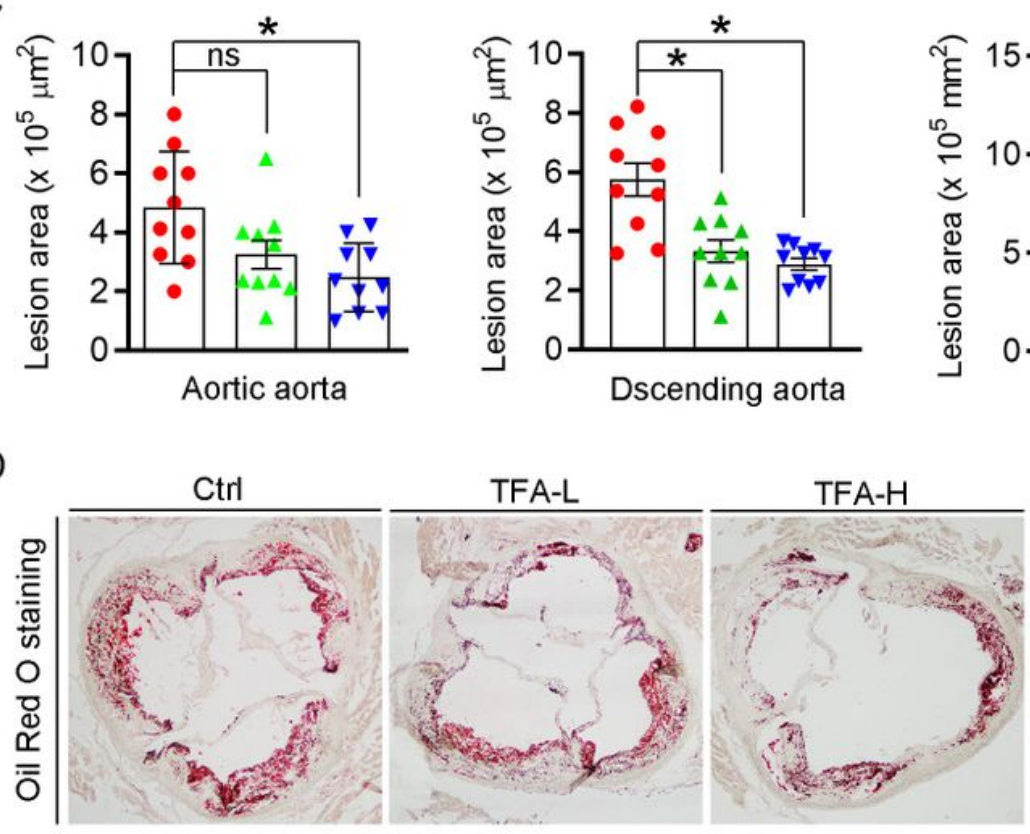

B
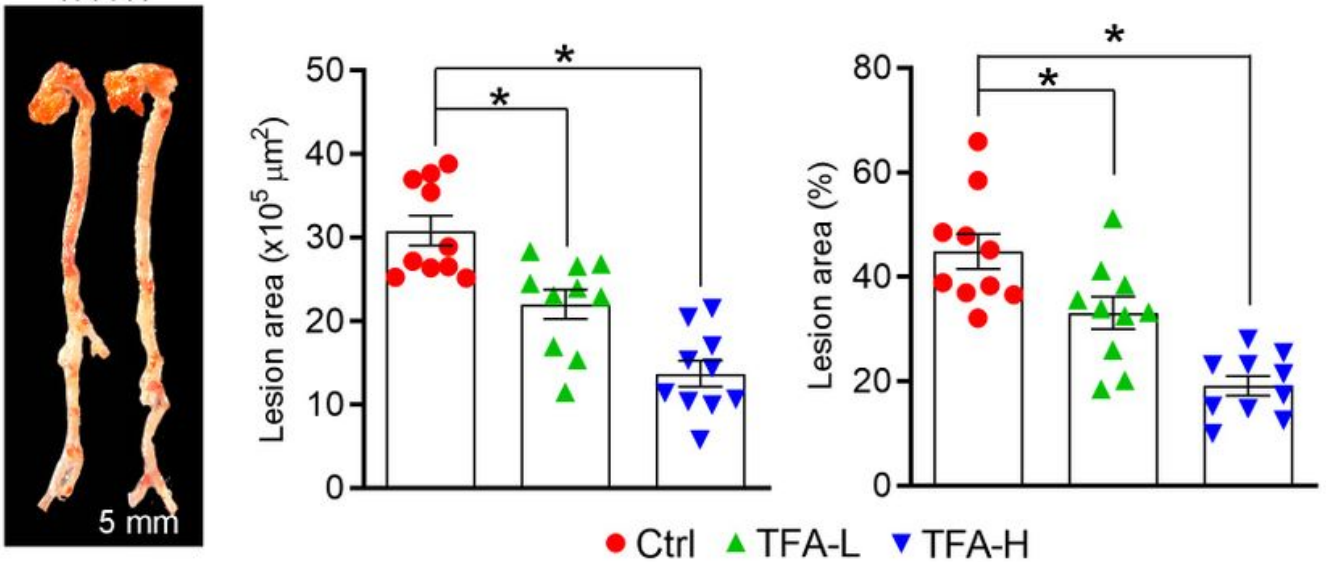
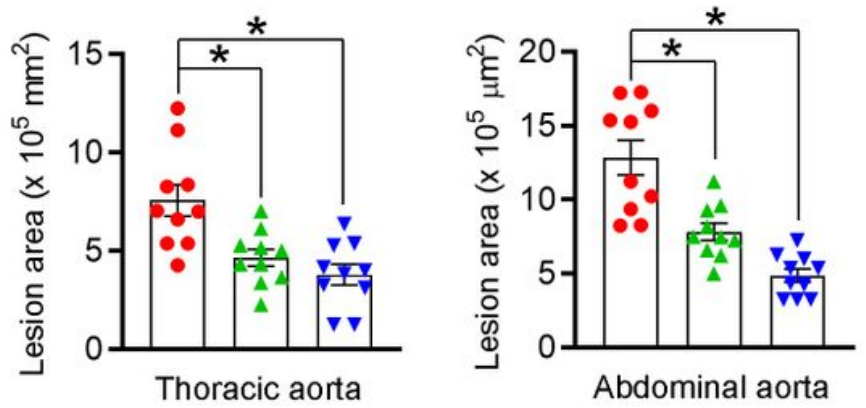

E
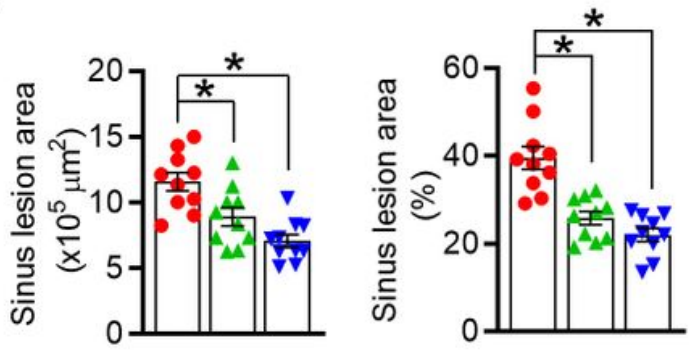

Figure 1 
TFA reduces atherosclerotic lesion size in apoE-/- mice. (A-B) Representative en face morphometric images of total aortic lesion area and calculated whole aortic atherosclerosis. Lesion areas were expressed as $\square \mathrm{m} 2$ or the ratio of lesion area to total area of aorta, $n=10$. (C) The lesion areas in different segments of aortas were also quantified. Lesion areas were expressed as $\square m 2, n=10$. (D-E) Lesions in aortic root cross sections were determined by Oil Red $O$ staining $(D)$ and quantified (E). Lesion areas were expressed as $\square \mathrm{m} 2$ or the ratio of lesion area to total area of aortic root cross sections, $n=10$. Data are presented as mean $\pm S E M, * P<0.05$, significantly different from control; ns: not significantly different.

Ma et al,Figure 2

A

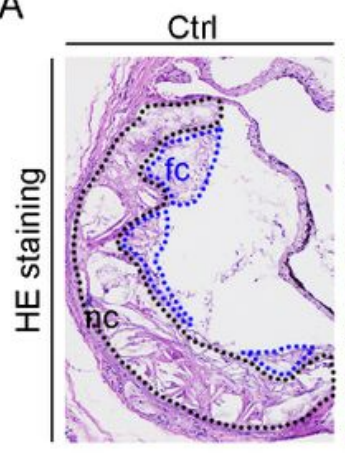

B

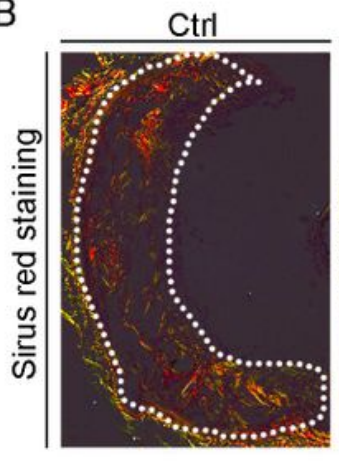

D

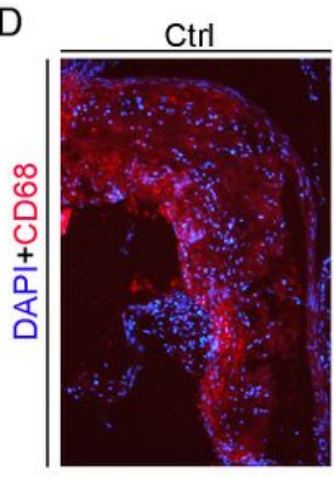

$\mathrm{F}$

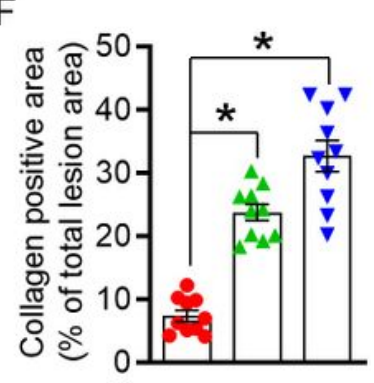

TFA-L
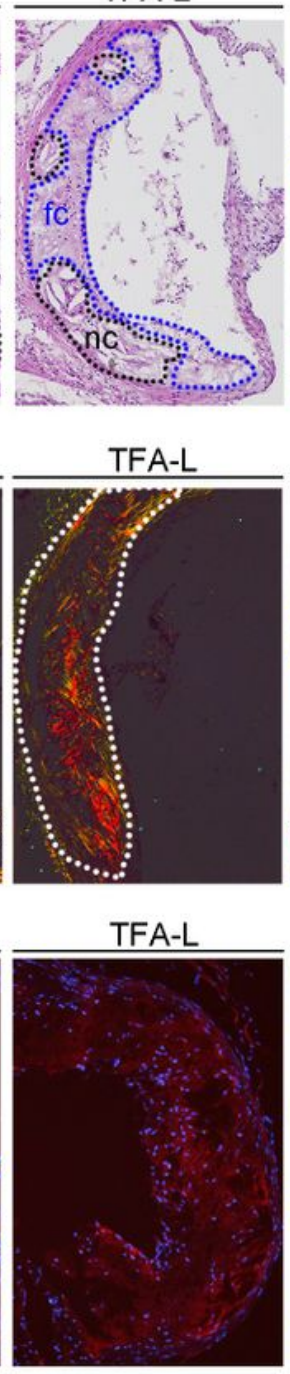

TFA-H

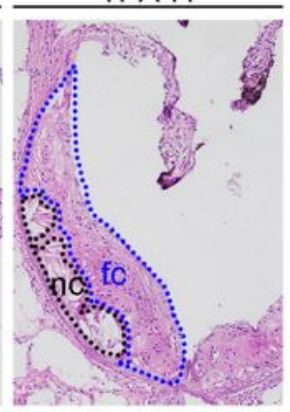

TFA-H

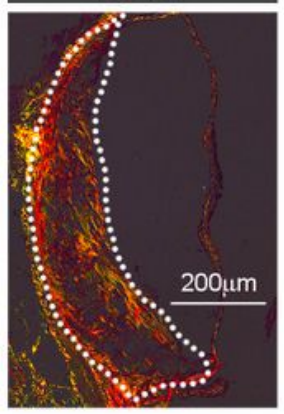

TFA-H

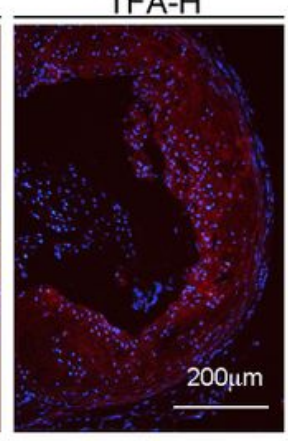

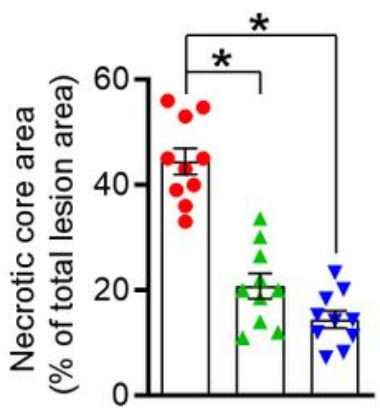

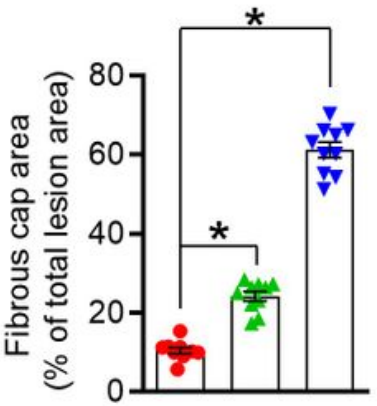

C

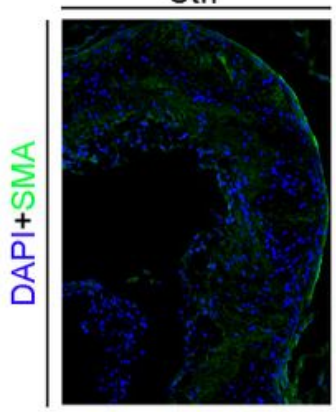

E

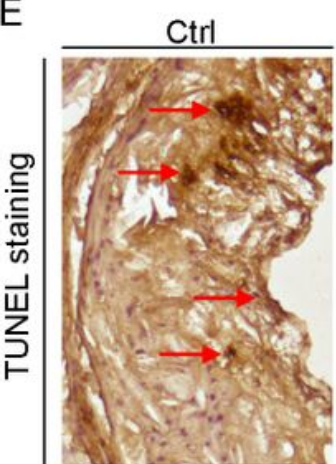

TFA-L

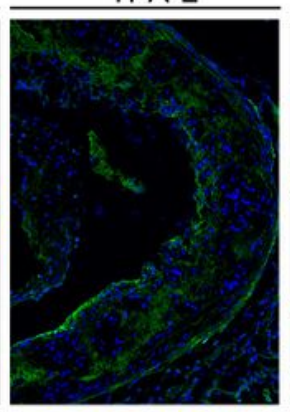

TFA-L

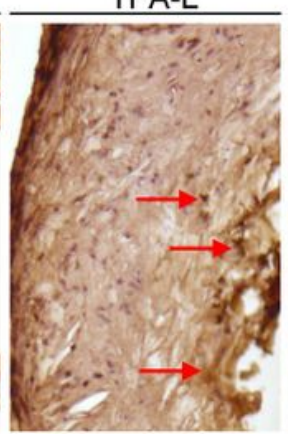

TFA-H

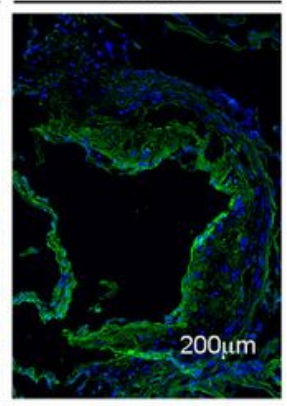

TFA-H

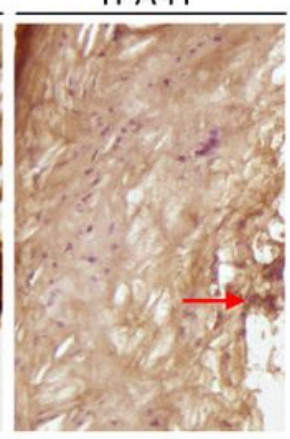

G




Figure 2

TFA changes the plaque composition and enhances plaque stability. (A) Haematoxylin and eosin staining followed by quantitative analysis of sinus lesions, necrotic core area, and fibrous cap area in aortic root cross sections. nc: necrotic cores; fc: fibrous cap, $n=10$. (B-F) Representative photomicrographs of aortic root sections stained with sirus red staining (B), aSMA (C), CD68 (D) and TUNEL staining (E) in atherosclerotic plaque followed by the quantification $(F), n=10$. (G) Vulnerability index of plaques, $n=10$. Data are presented as mean $\pm S E M,{ }^{*} P<0.05$, significantly different from control; ns: not significantly different.

Ma et al,Figure 3

A

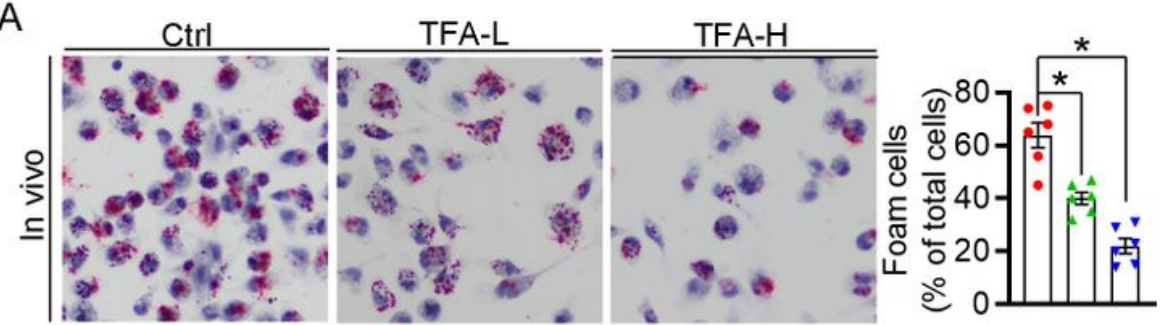

C

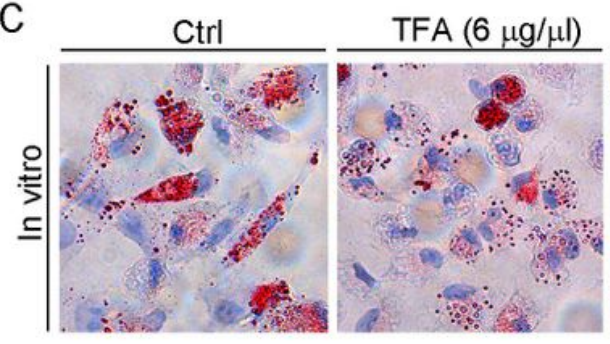

E

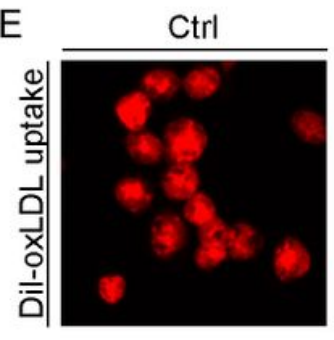

G
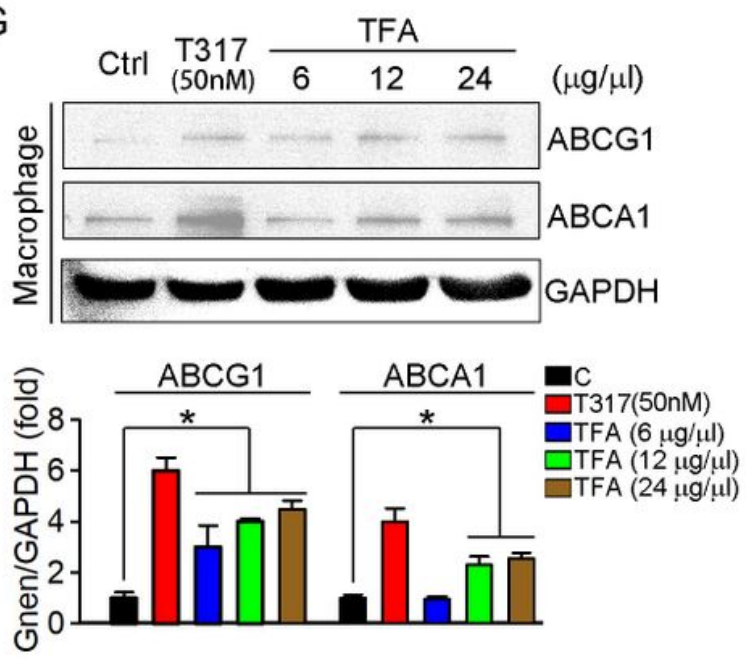

B

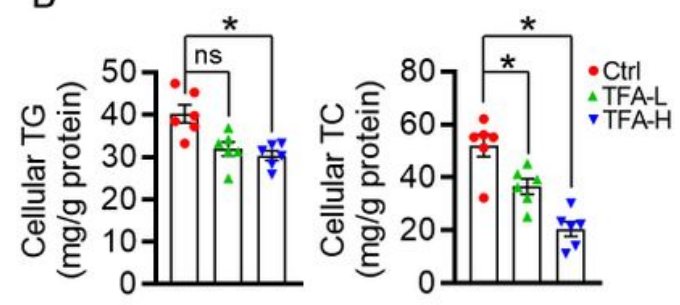

D
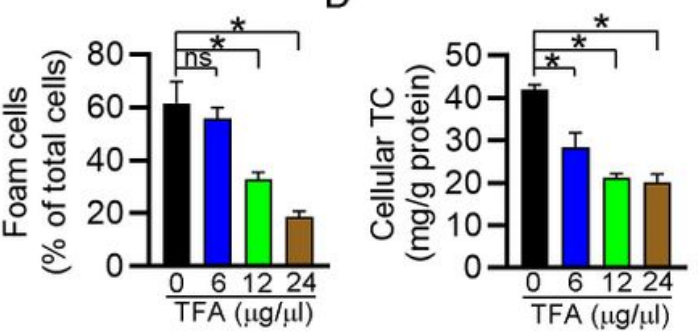

$\mathrm{F}$
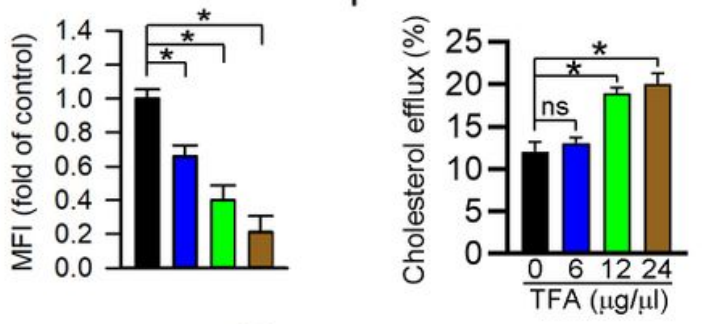

$\mathrm{H}$
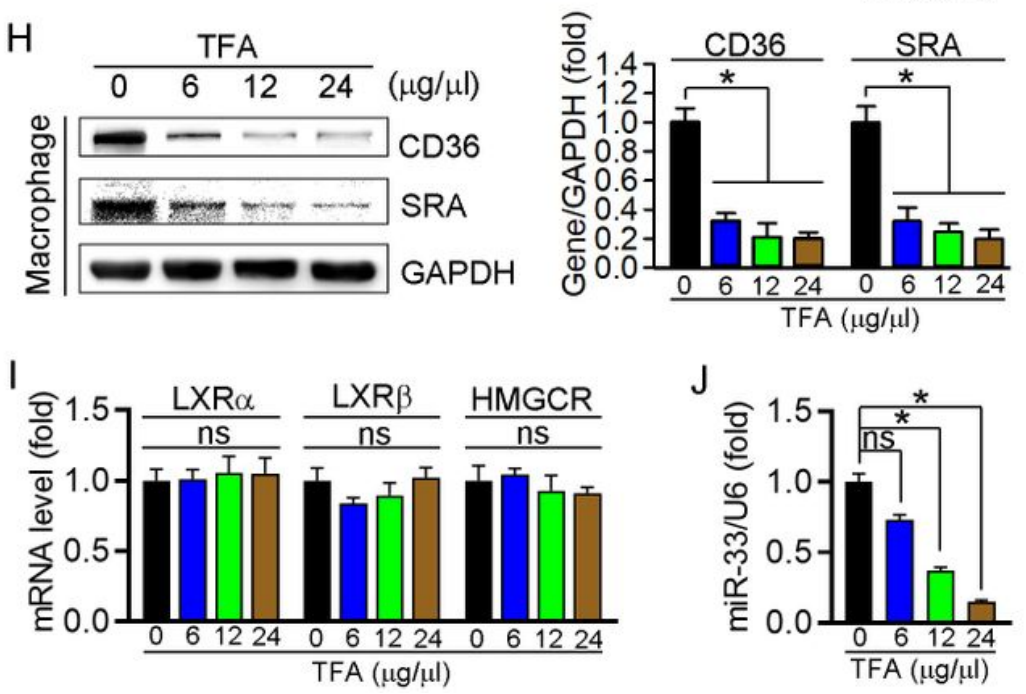

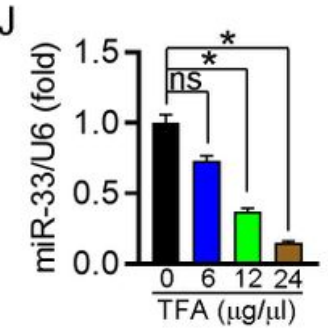

Figure 3 
TFA inhibits lipid accumulation and reduces expression of miR-33, CD36 and SRA in macrophage. (A) Inhibitory effect of TFA on foam cell formation using Oil Red 0 staining, $n=5$. (B) Determination of cellular TG and TC in peritoneal macrophage from apoE-/- mice, $n=5$. (C) Inhibitory effect of TFA on lipid accumulation using Oil Red $O$ staining in vitro, $n=5$. (D) Determination of cellular TC in RAW264.7 cells, $n=5$. (E) LDL uptake assay, $n=5$. (F) Cholesterol efflux assay, $n=5$. (G-H) Expression of ABCA1, ABCG1, CD36, and SRA was determined by western blot, $n=5$. (I) Expression of LXR囚, LXR囚囚 and HMGCR was determined by $q-R T-P C R, n=5$. $(J)$ Expression of miR-33 was determined by $q-R T-P C R, n=5$. Data are presented as mean $\pm S E M,{ }^{*} P<0.05$, significantly different from control; ns: not significantly different. TC: total cholesterol; TG: triglyceride; T317: T0901317, a synthetic ligand of LXR as a positive control.

A

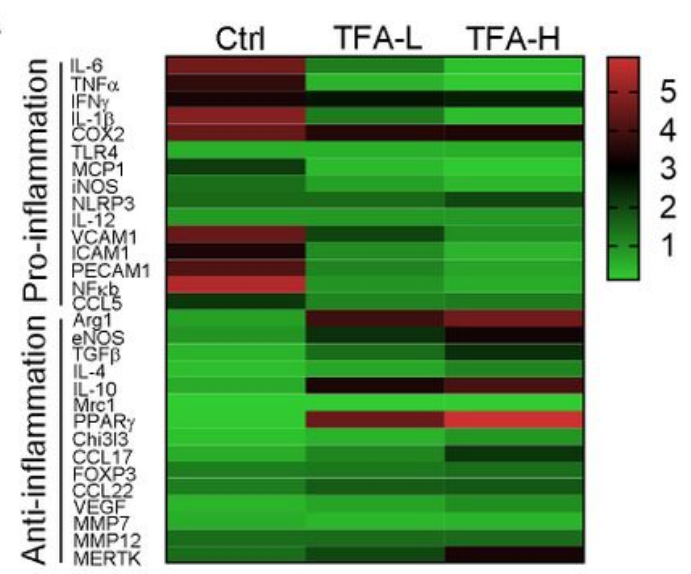

C
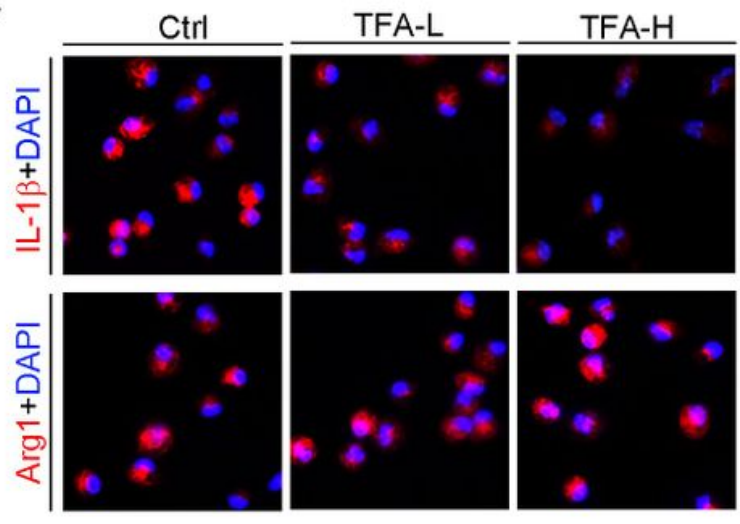

Ma et al,Figure 4
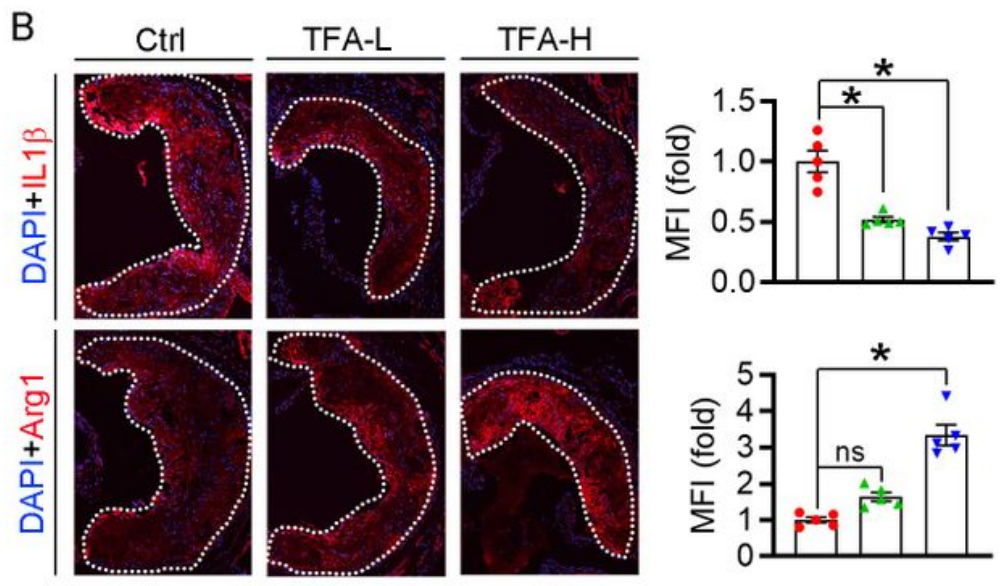

D
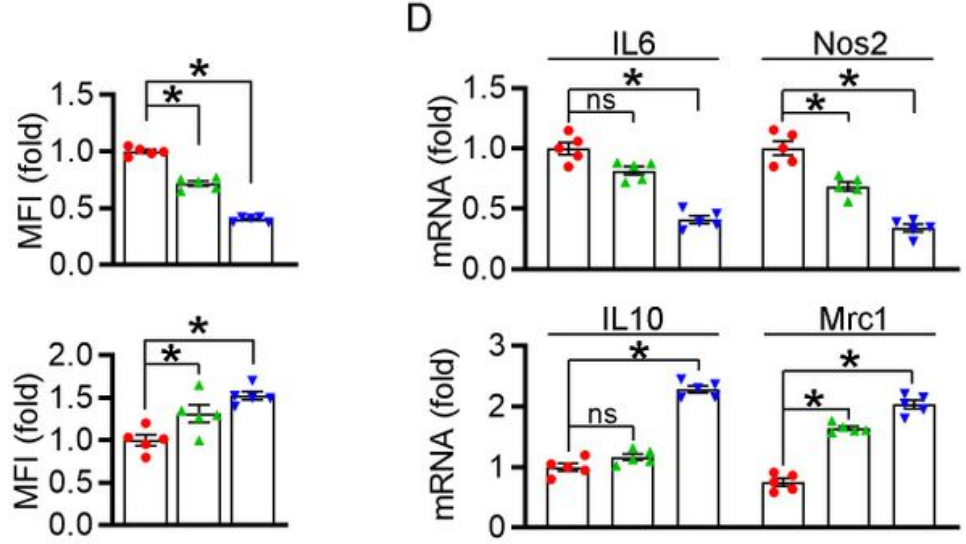

$E$
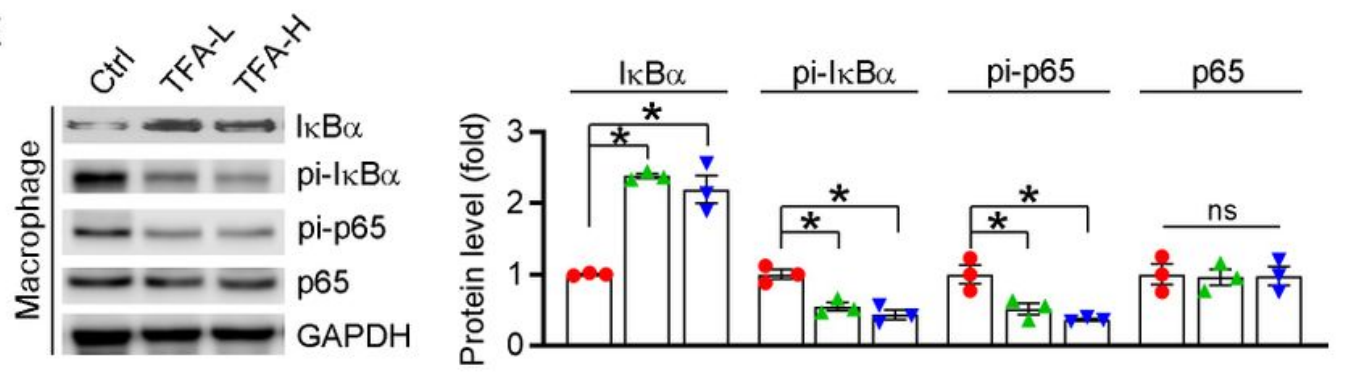

$\mathrm{F}$

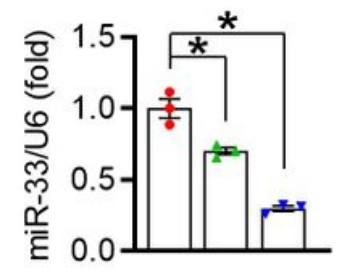

\section{Figure 4}

TFA reduces inflammatory response in vivo. (A) Transcriptional level of atherosclerosis-associated factors was assessed by q-RT-PCR, and the results were shown in heatmap, $n=3$. (B) Representative pro- 
and anti-inflammatory factors in the plaque was assessed by immunofluorescent staining, $n=5$. (C) Representative pro- and anti-inflammatory factors in peritoneal macrophage were assessed by immunofluorescent staining, n=5. (D) Expression of IL10, Nos2, Mrc1, and IL6 in peritoneal macrophage

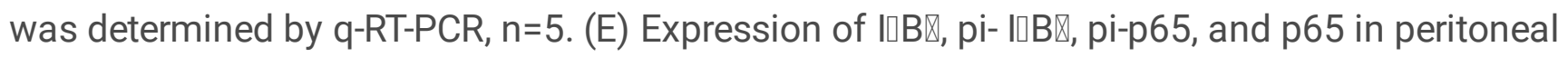
macrophage was determined by western blot, $n=3$. ( $F$ ) Expression of miR-33 in peritoneal macrophage from TFA-treated mice was determined by $q-R T-P C R, n=3$. Data are presented as mean $\pm S E M,{ }^{*} P<0.05$, significantly different from control; ns: not significantly different.

Ma et al,Figure 5

A
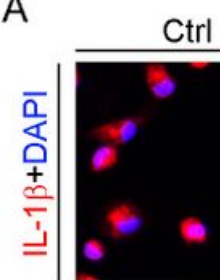
TFA $(6 \mu \mathrm{g} / \mu \mathrm{l}) \quad$ TFA $(12 \mu \mathrm{g} / \mu \mathrm{l}) \quad$ TFA $(24 \mu \mathrm{g} / \mu \mathrm{l})$
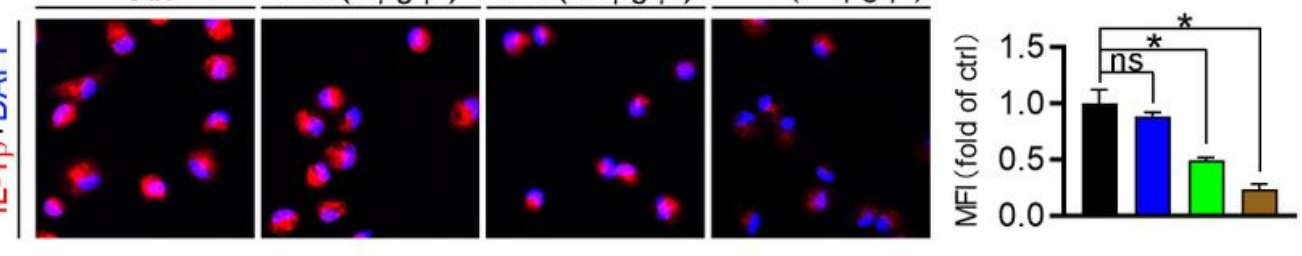

B
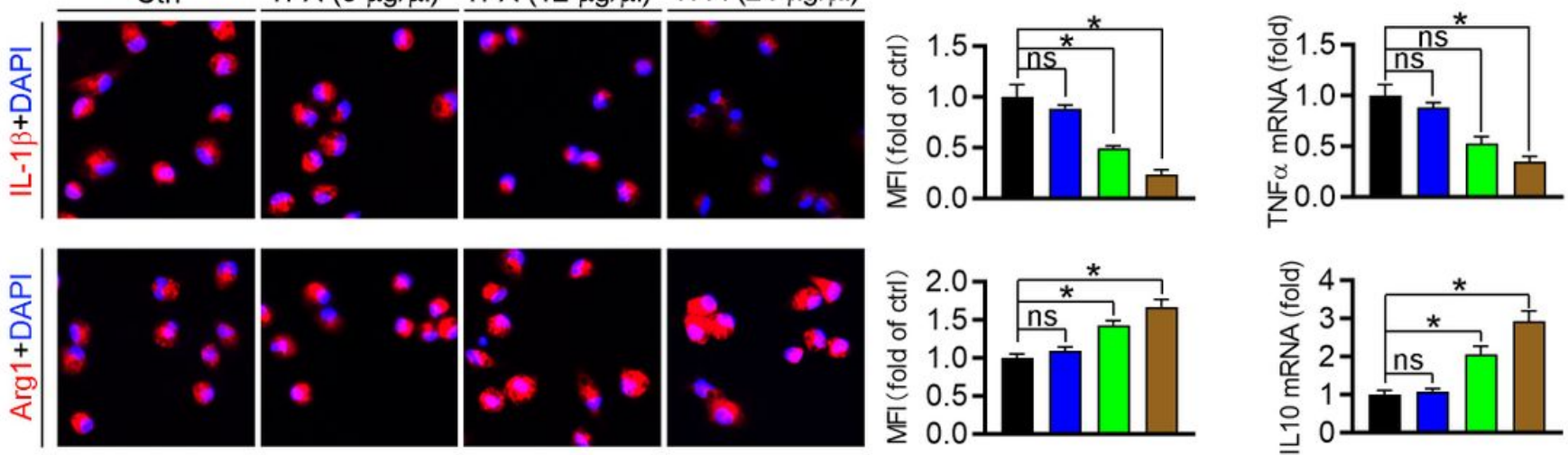

C
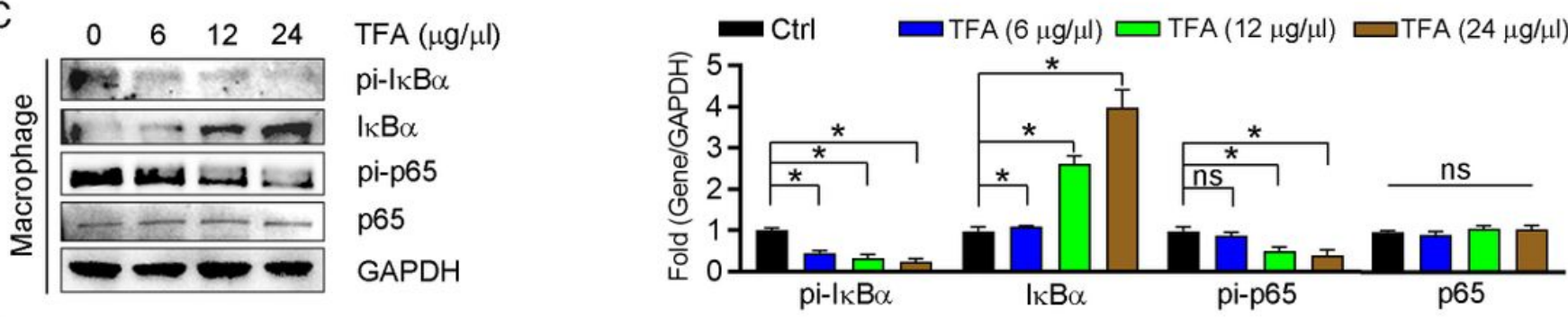

D
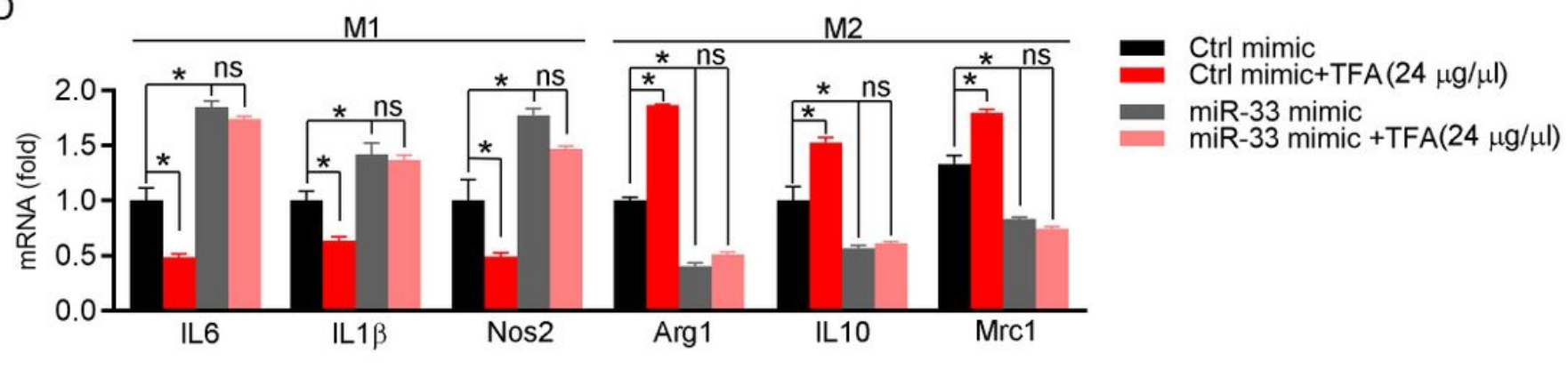

Figure 5

TFA inhibits inflammation and promotes the macrophage M2 transition. (A) After TFA treatment in peritoneal macrophage, expression of Arg1 and IL-1区 was determined by immunofluorescent staining, $\mathrm{n}=5$. (B) Expression of TNF囚 and IL-10 mRNA was determined by q-RT-PCR, $n=5$. (C) Expression of IkB IkBQ, pi-p65, and p65 was determined by western blot, $n=5$. (D) Expression of M1 or M2 markers was determined by $q-R T-P C R$ after treatment with miR-33 mimics and TFA, $n=5$. Data are presented as mean \pm $S E M, * P<0.05$, significantly different as indicated; ns: not significantly different. 
A

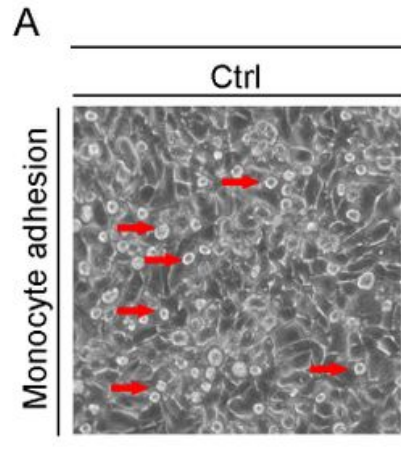

$\mathrm{C}$
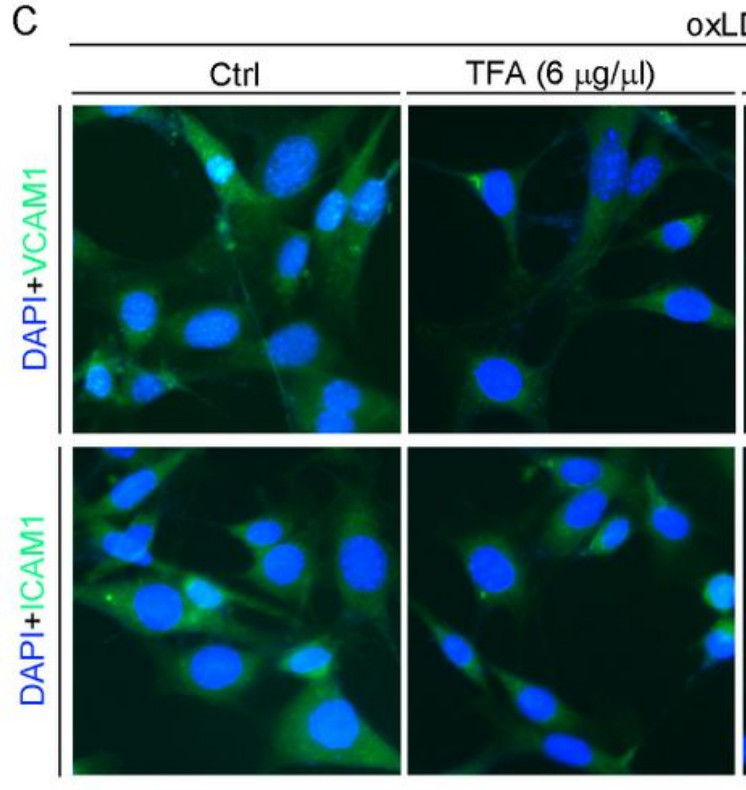

$\mathrm{E}$

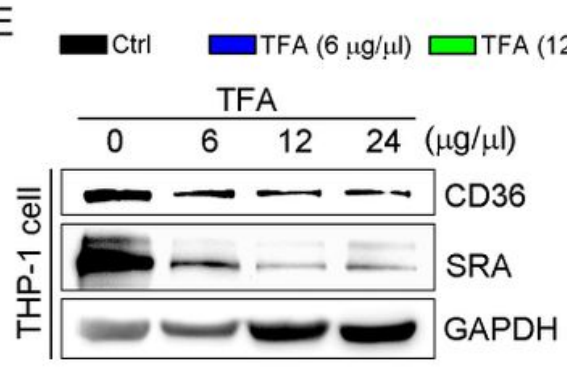

oxLDL

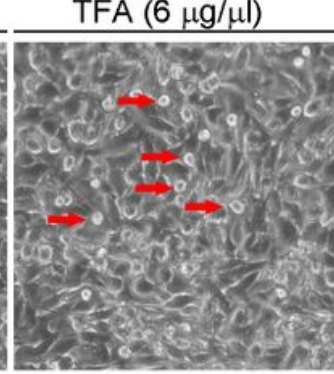

TFA $(12 \mu \mathrm{g} / \mu \mathrm{l})$

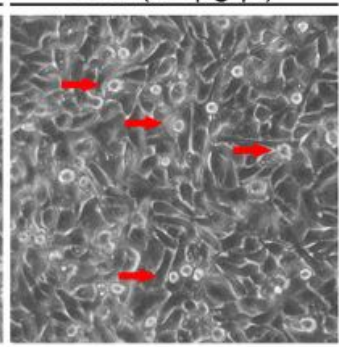

oxLDL

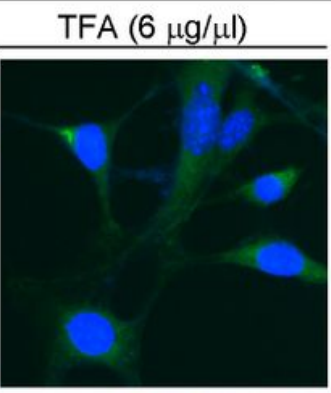

\section{TFA $(12 \mu \mathrm{g} / \mu \mathrm{l})$}
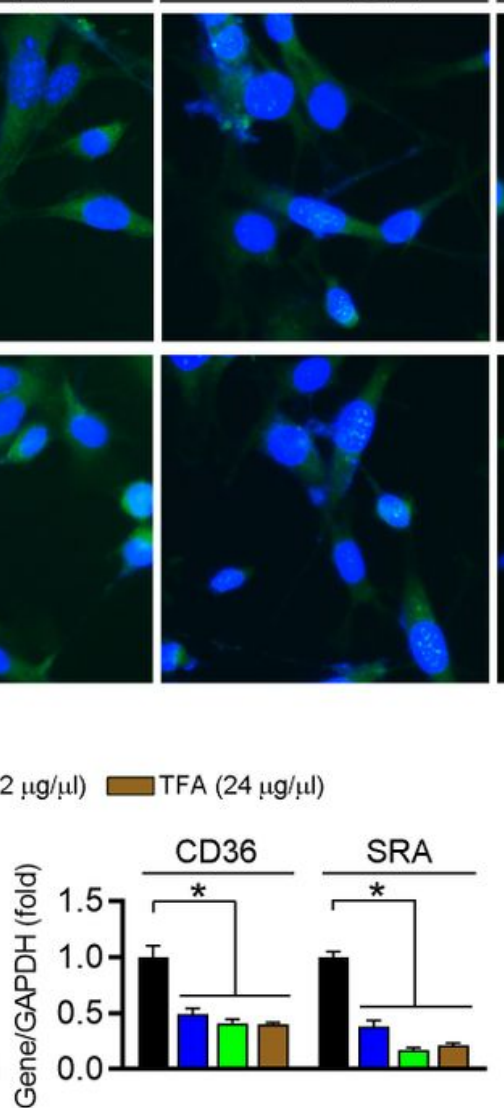

B

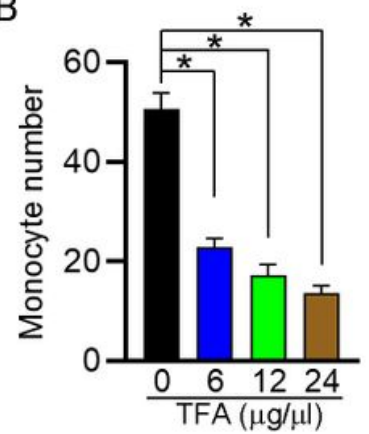

D
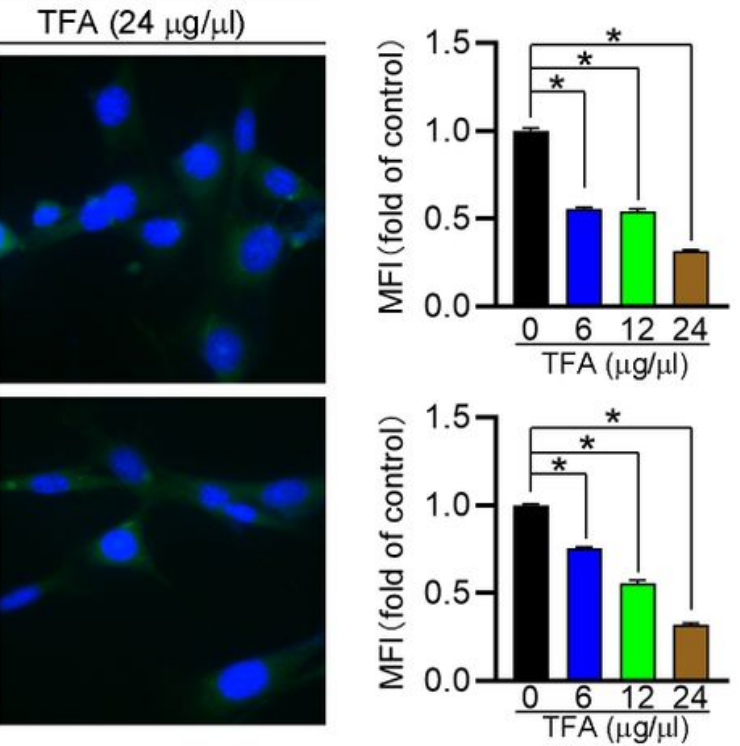

F

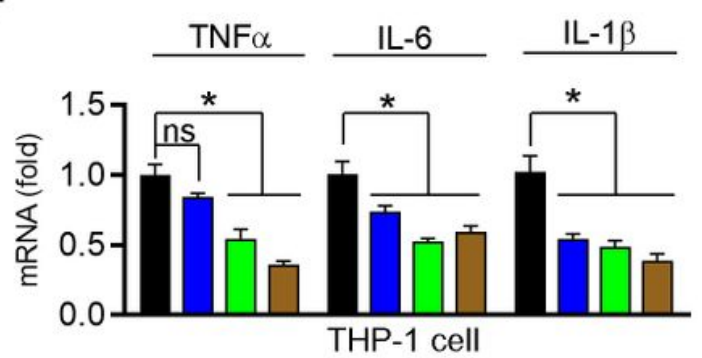

\section{Figure 6}

TFA inhibits monocyte adhesion to ECs. (A, B) HUVECs in 24-well plates and THP-1 cells were pretreated with oxLDL (100 $\mathrm{gg} \mathrm{mL-1)}$ for $2 \mathrm{~h}$. After incubation with oxLDL, THP-1 cells were added to HUVECs and co-incubated for $1 \mathrm{~h}$. The image of adherent THP-1 cells were captured with a microscope and the number of adherent THP-1 cells was calculated, n=5. (C, D) After TFA treatment in HUVEC, expression of ICAM-1 and VCAM-1 was determined by immunofluorescent staining, $n=5$. (E) Expression of CD36 and SRA protein in THP-1 cells was determined by western blot after treatment with TFA, $n=3$. (F) Expression of proinflammatory cytokines was determined by q-RT-PCR after treatment with TFA, $n=5$. Data are presented as mean $\pm \mathrm{SEM}$, ${ }^{\star} \mathrm{P}<0.05$, significantly different as indicated. 
Ma et al,Figure 7

A

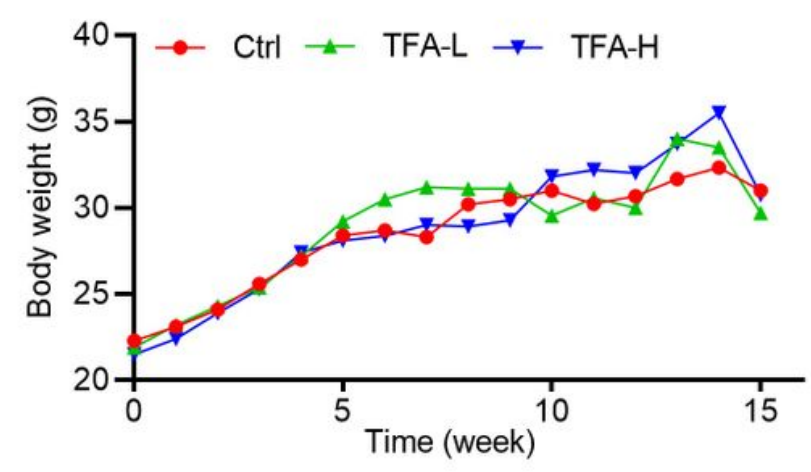

C

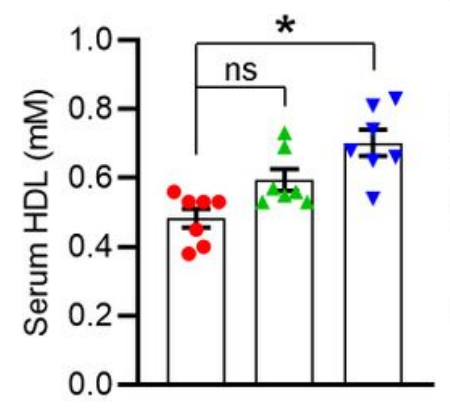

D

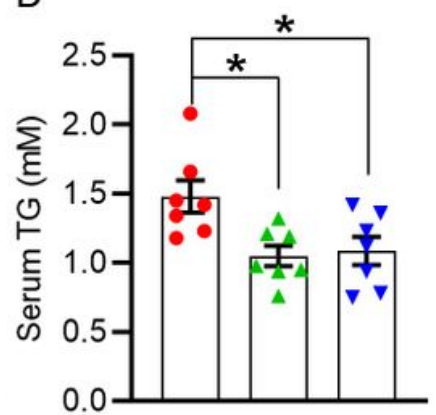

B

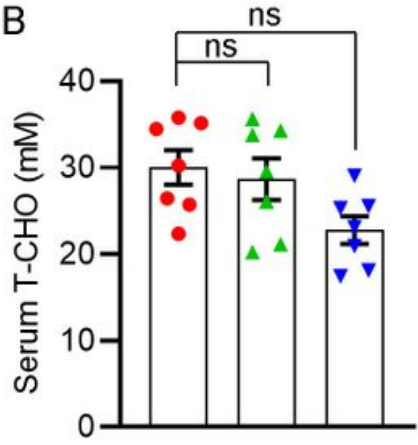

$\mathrm{E}$

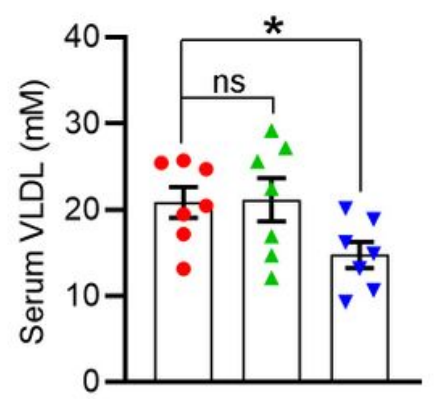

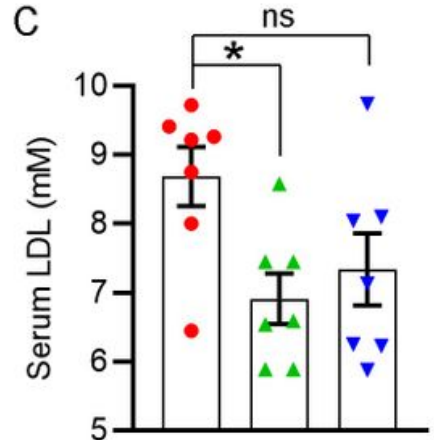

$\mathrm{F}$

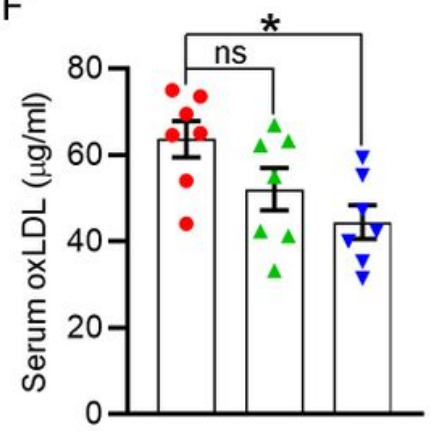

Figure 7

TFA improves the lipid disorder in vivo under HFD condition. (A) Body weight was recorded weekly. Date are presented as mean \pm SEM $(n=10)(B-F)$ Serum levels of total cholesterol (T-CHO), LDL- and HDL-C, VLDL, TG $(\mathrm{mM})$ and oxLDL $(\square \mathrm{g} / \mathrm{ml})$ were determined by biochemical analyzer or ELISA assay. Date are presented as mean \pm SEM $(n=7),{ }^{*}<0.05$, significantly different from control; $n s$ : not significantly different. 
Ma et al,Figure 8

A

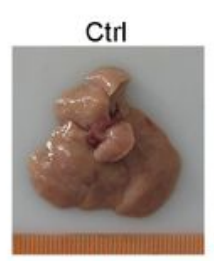

C

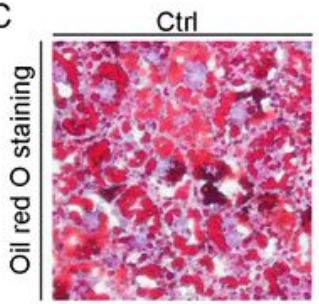

$\mathrm{F}$

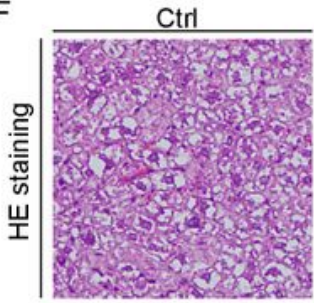

Ctrl

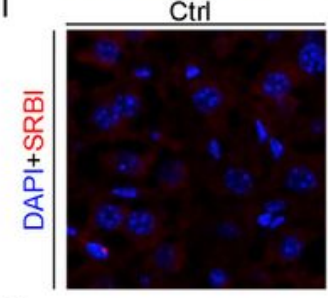

K

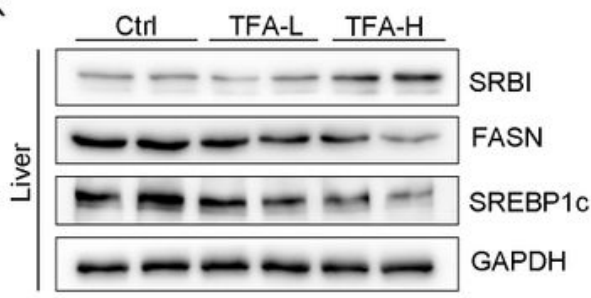

L
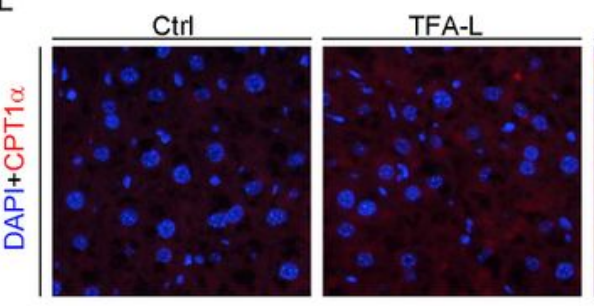

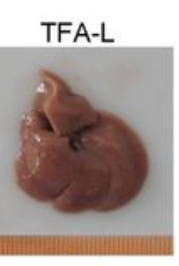

TFA-L

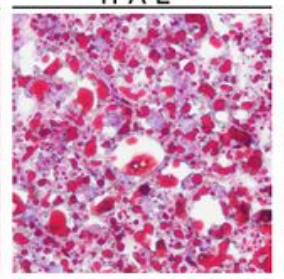

TFA-L

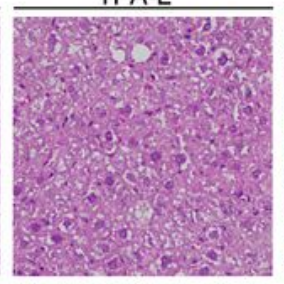

TFA-L
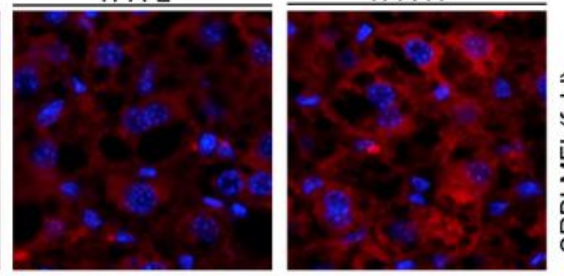

B

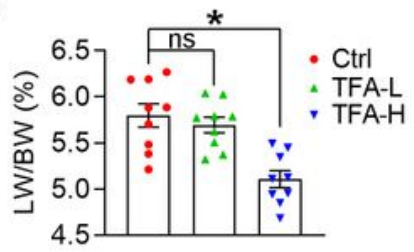

E

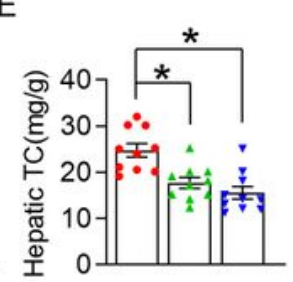

$\mathrm{H}$

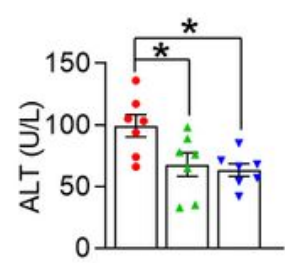

TFA-H

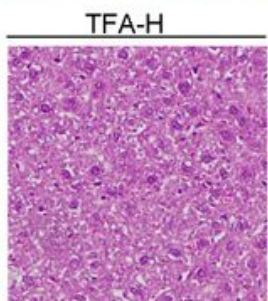

G

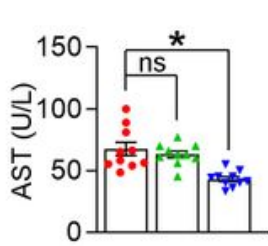

J

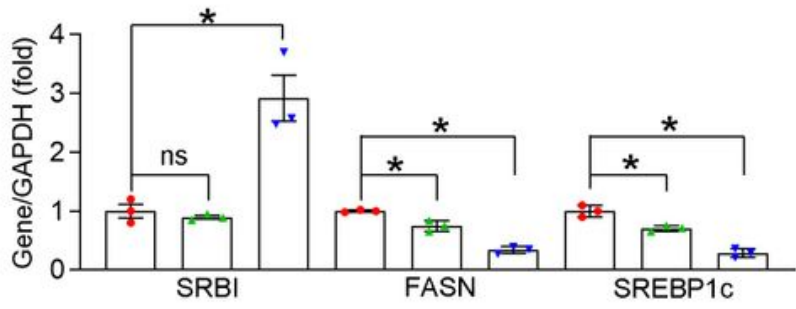

TFA-H

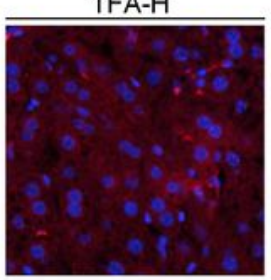

M
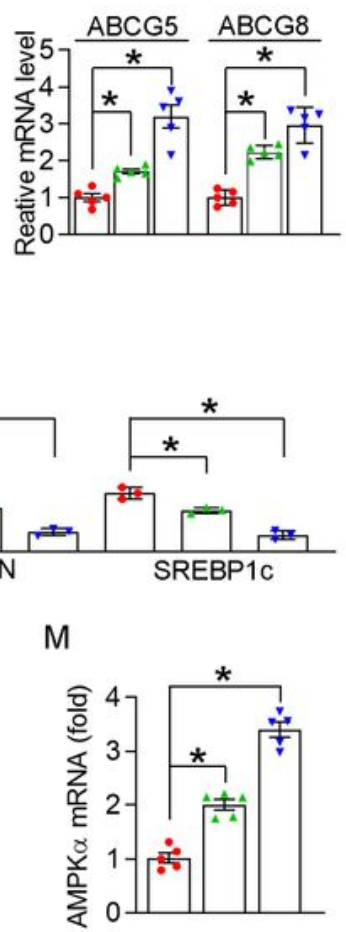

\section{Figure 8}

TFA ameliorates hepatic steatosis in vivo. (A) Liver photos and weight; (B) ratio of liver to body weight; (C) Oil Red O staining on liver frozen sections; (D-E) Quantitative analysis of hepatic TG and TC; (F) Haematoxylin and eosin staining of liver frozen sections; (G-H) Serum levels of AST and ALT; (I) Expression of SRBI was determined by immunofluorescent staining, $n=5$. (J) Expression of ABCG5 and ABCG8 was determined by q-RT-PCR, $n=5$. (K) Expression of SRBI, FASN, and SREBP1c, was determined 
by western blot, $n=3$. (L) Expression of $\mathrm{CPT} 1 \otimes$ was determined by immunofluorescent staining, $n=5$. (M) Expression of AMPK\ was determined by $q-R T-P C R, n=5$. Data are presented as mean $\pm S E M,{ }^{*} P<0.05$, significantly different as indicated.

\section{Supplementary Files}

This is a list of supplementary files associated with this preprint. Click to download.

- Supplementarydata.docx 